\title{
Auto-Regressive Model Based Polarimetric Adaptive Detection Scheme Part I: Theoretical Derivation and Performance Analysis
}

\author{
Fabiola Colone, Senior Member, IEEE, and Francesca Filippini, Member, IEEE
}

\begin{abstract}
This paper deals with the problem of target detection in coherent radar systems exploiting polarimetric diversity. We resort to a parametric approach and we model the disturbance affecting the data as a multi-channel autoregressive (AR) process. Following this model, a new polarimetric adaptive detector is derived, which aims at improving the target detection capability while relaxing the requirements on the training data size and the computational burden with respect to existing solutions. A complete theoretical characterization of the asymptotic performance of the derived detector is provided, using two different target fluctuation models. The effectiveness of the proposed approach is shown against simulated data, in comparison with alternative existing solutions.
\end{abstract}

Index Terms - polarimetric radar, adaptive signal detection, parametric approach, multi-channel auto-regressive process

\section{INTRODUCTION}

$\mathrm{P}$ OLARIZATION diversity has been widely employed to improve radar systems performance, see e.g. [1]-[28] and the reference therein. The additional information conveyed by different polarimetric channels can be fruitfully exploited in order to improve the system detection, recognition and classification performance. A multitude of works in the open literature have considered the use of multiple polarizations for a variety of applications, such as MIMO radar [7]-[11] , weather radar [12]-[14], or imaging radar [15]-[19], among others. More recently, the exploitation of such diversity has been considered for target classification based on the polarimetric micro-Doppler signature [20]-[23] as well as an effective way to improve the performance of passive radar systems [24]-[28].

With particular reference to the target detection stage, a number of detectors that exploit a set of polarimetric-temporal (or polarimetric-temporal-spatial) observations has been conceived and extensively studied, e.g. [4]-[9]. Traditional approaches to this problem devised for coherent polarimetric radar systems operating against Gaussian disturbance components are fully adaptive polarimetric detectors, such as the polarimetric generalized likelihood ratio test (GLRT) in [4]-[5] and the polarimetric adaptive matched filter (AMF) in [6].

F. Colone and F. Filippini are with the Dept. of Information Engineering, Electronics and Telecommunications Sapienza University of Rome, 00184, Italy, (e-mail: fabiola.colone@uniroma1.it, francesca.filippini@uniroma1.it) (Corresponding author: F. Colone)
These methods exploit all the available degrees of freedom to perform disturbance cancellation without requiring any underlying spectral model. Therefore, they are based on the availability of a large amount of target-free training data, that allow to estimate and invert the unknown disturbance covariance matrix. In fact, a number of secondary data that is at least twice the size of the data is needed for the adaptive detector to be used. This requirement becomes an issue in applications where the number of adaptive degrees of freedom is large, as well as in heterogeneous environment where the available secondary data are not representative of the disturbance in the cell under test. In such cases, the requirement on such large amount on training data typically cannot be met, thus preventing the conventional adaptive polarimetric detectors to be used.

To alleviate the training data requirement, several approaches have been pursued in the open literature leveraging some prior knowledge about the disturbance covariance matrix, see e.g. [29]-[37] and the references therein. Among them, the authors in [30] and [31] exploit the Toeplitz structure to derive a covariance matrix estimator and the corresponding radar detector. The persymmetric structure of the interference covariance matrix is exploited in [32]-[35], to derive a maximum likelihood (ML) estimate [32], and corresponding detectors for both Gaussian data [33],[34] and for non-Gaussian clutter modelled by spherically invariant random vectors [34],[35]. In [36], a ML estimator is derived when a constraint on the condition number is imposed, modelling the disturbance as a sum of colored interference plus white disturbance; in [37] a rank-constrained ML estimator is developed.

Another way to effectively address this issue and reduce the computational burden is to resort to parametric (or modelbased) approaches [38]-[54]. Specifically, parametric approaches have been first employed in single-channel radar systems [41]-[46], where only the temporal domain is exploited for clutter cancellation and target detection. Later on, model-based strategies have been extended to multichannel radar systems [47]-[54]. In this framework, the possibility to model the disturbance contribution as a multichannel autoregressive (AR) process and exploit it for the target detection has been proved to be a successful solution, useful in a multitude of applications, such as space-time adaptive processing (STAP) [49]-[54]. 
In this work, we resort to this approach for radar systems equipped with multiple polarimetric channels and we develop an effective solution that aims at improving the target detection capability while relaxing the requirement on the training data size and the computational burden. Specifically, we design a polarimetric adaptive detection scheme based upon a two-stage procedure: first, we derive the GLRT based upon the primary data only, assuming that the disturbance characteristics are a priori known, then we make the derived detector fully adaptive by substituting the maximum likelihood estimation of the disturbance parameters, based upon a set of target-free training data, into the clairvoyant detector in place of its unknown values. Consequently, the derived detector will be referred to as AR model based polarimetric adaptive matched filter.

For the derivation of the proposed detector, we use a partially structured model for the target, namely we assume that the target returns are known up to an unknown amplitude in the temporal domain, but they involve unknown nonlinear signal parameters in the polarimetric domain. This assumption, which will be further discussed in Section II, makes the devised detector different from existing parametric approaches previously proposed for multi-channel radar systems, where typically a fully structured model is used for the target component, e.g. [49],[52]. The general case of a multi-rank subspace target model was recently considered in [54] where a subspace parametric Rao test has been used to handle the detection problem. However, in this work, the target component has a special structure which is peculiar of polarimetric radar. In this case, a GLRT approach can be used to derive the sought detector. Consequently, a dedicated performance characterization is also required.

In Part I of this two-part study, we derive the proposed polarimetric adaptive target detector and we present closedform mathematical expressions for its asymptotic performance, both assuming a non-fluctuating and fluctuating model for the target echoes to be detected. Moreover, we carry out an extensive analysis of its performance against simulated data for training sets of finite size. The reported analyses prove the superiority of the proposed detection scheme when a limited number of secondary data is employed. Throughout this work we assume that the input disturbance process exactly matches the multi-channel AR model exploited for the derivation of the proposed detector. The case of an input disturbance that does not match the adopted spectral model will be the object of the companion paper [55]. In [55], also the application of the proposed scheme against experimental data is considered following the encouraging preliminary results obtained in [56] for the case of a passive radar system.

The remainder of this manuscript is organized as follows. In Section II prior work on polarimetric adaptive detector is detailed. The proposed AR model based polarimetric adaptive detection scheme is derived in Section III. The asymptotic performance of the proposed detector is illustrated in Section IV while an extensive numerical analysis is reported in Section $\mathrm{V}$ to assess the performance of the derived detection strategy. Finally, concluding remarks are given in Section VI while mathematical details are reported in the Appendices.

Notation: Throughout this manuscript, matrices (vectors) are set in boldface, with uppercase (lowercase) letters. The subscripts $(\cdot)^{T},(\cdot)^{*}$ and $(\cdot)^{H}$ mean transpose, conjugate and Hermitian (or conjugate transpose), respectively. The Kronecker product is denoted by $\otimes$ while $\mathrm{u}(\cdot)$ indicates the unit step function. Identity and null $(N \times N)$ matrices are denoted by $\mathbf{I}_{N}$ and $\mathbf{0}_{N}$, respectively while $\mathbf{0}_{N \times M}$ indicates the null $(N \times M)$ matrix. $|\cdot|$ means determinant for matrices and modulus for scalars while $\operatorname{tr}(\cdot)$ denotes the trace of a matrix. $\mathfrak{R}(\cdot)$ and $\mathfrak{I}(\cdot)$ indicate the real and imaginary part, respectively and $\mathcal{C} \mathcal{N}(\mathbf{v}, \mathbf{N})$ denotes the multivariate complex Gaussian distribution with mean vector $\mathbf{v}$ and covariance matrix $\mathbf{N}$. The expectation operator is indicated by $E\{\cdot\}$.

\section{Fully AdAPTIVE POLARIMETRIC DETECTORS}

Let us consider a polarimetric radar system that collects signals from $L$ polarimetric channels. These are typically obtained by exploiting multiple receiving channels connected to differently polarized antenna elements. A quite typical case is attained by exploiting two linearly cross-polarized antennas, making $L=2$ polarimetric channels available. The number of equivalent polarimetric channels can be further increased if the transmitter is able to emit signals using different polarizations. For instance, by alternatively transmitting bursts of radar pulses at the two linear polarizations ( $\mathrm{H}$ and $\mathrm{V}$ ) and simultaneously collecting the corresponding backscattered echoes at both polarizations, a maximum of $L=4$ polarimetric channels can be obtained in a pulse radar, namely $\mathrm{HH}, \mathrm{VV}, \mathrm{HV}$ and $\mathrm{VH}$ channels. Assuming reciprocity between $\mathrm{HV}$ and $\mathrm{VH}$ returns [2], whose compliance could be verified using the approach proposed in [57], the number of independent channels is $L=3$.

For a generic $L$ value, we arrange in a $L$-dimensional vector $\mathbf{x}_{0}(m)$ the samples collected at the available polarimetric channels at the $m$-th temporal observation:

$$
\mathbf{x}_{0}(m)=\left[x_{0}^{(0)}(m) \ldots x_{0}^{(1)}(m) \ldots x_{0}^{(L-1)}(m)\right]^{T}
$$

In this paper, temporal observations might refer to consecutive pulses emitted by a pulse radar transmitter; in this case the samples are extracted at a given range cell after an appropriate range compression stage, e.g. matched filter. Alternatively, they might refer to samples of the backscattered echo signal for a continuous wave radar transmission. The subscript ' 0 ' in (1) refers to primary data, namely the data in which the detection of a target echo is sought. In fact, $\mathbf{x}_{0}(\mathrm{~m})$ is the sum of a useful target component $\mathbf{s}(m)$ and a vector $\mathbf{d}(m)$ collecting the disturbance contributions, which might include clutter and noise:

$$
\mathbf{x}_{0}(m)=\gamma \mathbf{s}(m)+\mathbf{d}(m)
$$


being $\gamma=0$ under the null hypothesis $H_{0}$ (target absent) and $\gamma=1$ under the alternate $H_{1}$ hypothesis (target present).

We arrange $M$ consecutive temporal observations in a $L M \times 1$ vector

$$
\mathbf{x}_{0}=\left[\mathbf{x}_{0}^{H}(0) \mathbf{x}_{0}^{H}(1) \ldots \mathbf{x}_{0}^{H}(M-1)\right]^{H}
$$

Following (2), it can be decomposed as $\mathbf{x}_{0}=\gamma \mathbf{s}+\mathbf{d}$, where $\mathbf{s}$ and $\mathbf{d}$ are arranged as $\mathbf{x}_{0}$. Specifically, the target component can be written as:

$$
\mathbf{s}=\mathbf{t} \otimes \boldsymbol{\alpha}
$$

where

- $\mathbf{t}$ is the vector of expected temporal returns for a unit amplitude target echo. It is assumed known at the receiver, however its definition depends on the employed radar system. For an active radar system exploiting a coherent train of $M$ pulses, $\mathbf{t}$ is typically referred to as the temporal steering vector and it is given by $\mathbf{t}=$ $\left[1, e^{-j 2 \pi f_{d}}, \ldots, e^{-j 2 \pi(M-1) f_{d}}\right]^{H}$, being $f_{d}$ the target Doppler frequency normalized to the pulse repetition frequency (PRF). In contrast, when a CW radar is considered and the temporal observations represent consecutive samples of the backscattered echo, $\mathbf{t}$ might include a $M$-samples fragment of the transmitted signal.

- $\boldsymbol{\alpha}=\left[\alpha_{0}, \ldots, \alpha_{L-1}\right]^{T}$ contains the unknown complex target amplitudes at the different polarimetric channels. As is apparent, a partially structured model is assumed for the target. In fact, its returns are assumed to be known up to an unknown amplitude in the temporal domain, namely at the $l$-th polarimetric channel, $l=0, \ldots, L-1$, they are obtained as $\alpha_{l} \mathbf{t}$, with known $\mathbf{t}$. In contrast, target returns involve unknown nonlinear parameters in the polarimetric domain, being the target echoes at a given time instant provided by $\mathrm{t}_{m} \boldsymbol{\alpha}, m=0, \ldots, M-1$, with unknown $\boldsymbol{\alpha}$.

Vector $\mathbf{x}_{0}$ is typically modelled as a complex Gaussian random vector with covariance matrix $\mathbf{M}$, zero mean vector under the null hypothesis $H_{0}$ and mean vector $\mathbf{s}$ under the alternate hypothesis $H_{1}$, i.e. $\left.\mathbf{x}_{0}\right|_{H_{\gamma}} \sim \mathcal{C N}(\gamma \mathbf{s}, \mathbf{M})$.

The $L M \times L M$ covariance matrix $\mathbf{M}$ encodes the polarimetric and spectral characteristics of the disturbance affecting the received vector and it has a block structure:

$$
\mathbf{M}=\left[\begin{array}{ccc}
\mathbf{M}_{0,0} & \cdots & \mathbf{M}_{0, M-1} \\
\vdots & \ddots & \vdots \\
\mathbf{M}_{M-1,0} & \cdots & \mathbf{M}_{M-1, M-1}
\end{array}\right]
$$

where the generic $L \times L$ block $\mathbf{M}_{m, p}$ is the cross-covariance matrix of the polarimetric vectors extracted at times $m$ and $p$, namely $\mathbf{M}_{m, p}=\left\{\mathbf{x}_{0}(m) \mathbf{x}_{0}^{H}(p) \mid H_{0}\right\}$.

As is well known, under these hypotheses and assuming the covariance matrix $\mathbf{M}$ known, the optimum detection test, referred to as the polarimetric matched filter (Pol-MF), is obtained as [6]

$$
T_{\text {Pol-MF }}=\mathbf{x}_{0}^{H} \mathbf{M}^{-1} \mathbf{T}\left[\mathbf{T}^{H} \mathbf{M}^{-1} \mathbf{T}\right]^{-1} \mathbf{T}^{H} \mathbf{M}^{-1} \mathbf{x}_{0} \underset{H_{0}}{\stackrel{H_{1}}{\gtrless}} \eta_{M F}
$$

where $\eta_{M F}$ is the detection threshold and $\mathbf{T}=\mathbf{t} \otimes \mathbf{I}_{L}$.

However, adaptive approaches must be considered to obtain a practical receiver for real radar scenarios where the covariance matrix is unknown. To this end, along with the primary data $\mathbf{x}_{0}$, the authors in [4]-[6] assume a set of $P$ vectors $\mathbf{x}_{p}, p=1, \ldots, P$, to be available, referred to as secondary (or training) data. They are assumed target-free, independent and identically distributed (i.i.d), sharing the same statistic of $\mathbf{x}_{0}$ under the $H_{0}$ hypothesis.

Two different adaptive detection schemes can be obtained by resorting to either a two-stage GLRT strategy or a plain GLRT approach.

Specifically, in the former case, the detection test is obtained by substituting in (5) the ML estimate of the covariance matrix based upon $P$ secondary data, namely $\widehat{\mathbf{M}}=$ $\frac{1}{P} \sum_{p=1}^{P} \mathbf{x}_{p} \mathbf{x}_{p}^{H}$, thus writing [6]:

$$
T_{\text {Pol-AMF }}=\mathbf{x}_{0}^{H} \widehat{\mathbf{M}}^{-1} \mathbf{T}\left[\mathbf{T}^{H} \widehat{\mathbf{M}}^{-1} \mathbf{T}\right]^{-1} \mathbf{T}^{H} \widehat{\mathbf{M}}^{-1} \mathbf{x}_{0} \underset{H_{1}}{\stackrel{H_{0}}{\gtrless} \eta_{A M F}}
$$

where $\eta_{A M F}$ is a properly modified detection threshold.

This will be referred in the following as the Polarimetric adaptive matched filter (Pol-AMF).

Alternatively, by resorting to the plain GLRT approach, the polarimetric GLRT (Pol-GLRT) has been derived in [4] and [5] as

$$
\begin{aligned}
T_{\text {Pol-GLRT }}= & \frac{\mathbf{x}_{0}^{H} \widehat{\mathbf{M}}^{-1} \mathbf{T}\left[\mathbf{T}^{H} \widehat{\mathbf{M}}^{-1} \mathbf{T}\right]^{-1} \mathbf{T}^{H} \widehat{\mathbf{M}}^{-1} \mathbf{x}_{0}}{P+\mathbf{x}_{0}^{H} \widehat{\mathbf{M}}^{-1} \mathbf{x}_{0}} \\
& =\frac{T_{P o l-A M F}}{P+\mathbf{x}_{0}^{H} \widehat{\mathbf{M}}^{-1} \mathbf{x}_{0}} \underset{H_{0}}{\gtrless} \eta_{G L R T}
\end{aligned}
$$

$\eta_{G L R T}$ being the detection threshold that guarantees the desired false alarm probability.

As it is apparent, the above detectors adaptively exploit all the available degrees of freedom to perform the whitening of the data $\mathbf{x}_{0}$ in both the polarimetric and temporal domains. While being theoretically optimum under the assumption of $a$ priori known disturbance characteristics, this approach might be computationally intensive and might suffer of significant adaptivity loss in practical cases, especially when a limited number of training data is made available. As is well known, a number of secondary data $P \geq 2 M L$ is required for the aforementioned adaptive detectors to yield limited adaptivity loss. This could result in severe limitations when $L$ and $M$ are large, as it may be difficult to obtain the required amount of training data with the desired characteristics (target-free and i.i.d). 
To overcome these issues, in the next Section we exploit a parametric method to develop a new polarimetric adaptive detector by modeling the disturbance as a multi-channels AR process.

\section{AR MODEL-BASED POLARIMETRIC ADAPTIVE DETECTOR}

One effective way to reduce the computational load and training data size requirements is to model the disturbance as a multi-channel AR process and exploit it for the development of the target detection test. This approach has been effectively applied in several radar applications including STAP and other array processing applications.

Here this approach is employed to the considered polarimetric radar where the multi-channel system is intended to capture the polarimetric information from both the target and the competing disturbance.

Specifically, the adopted disturbance model is described in sub-section III-A. This is then exploited in sub-section III-B to derive the GLRT detection test based on primary data only, namely by assuming known the disturbance parameters.

Finally, the AR-based adaptive detection test is presented in sub-section III-C, based on the result in III-B and a two-stage GLRT approach.

\section{A. Multi-channel Autoregressive Model}

We model the disturbance as a $L$-channel AR process of known order $(Q-1)$, denoted as $\operatorname{AR}(Q-1)$ and exploit it for signal detection.

Accordingly, the vector random process $\mathbf{d}(m)$ satisfies the following relation [38]:

$$
\mathbf{d}(m)=\sum_{q=1}^{Q-1} \mathbf{A}^{H}(q) \mathbf{d}(m-q)+\mathbf{w}(m)
$$

where $\{\mathbf{A}(q)\}_{q=1}^{Q-1}$ are complex-valued $L \times L$ matrix parameters encoding the regression coefficients at different polarimetric channels, and $\mathbf{w}(m) \sim C N(\mathbf{0}, \mathbf{R})$ is the driving white noise sequence, $\mathbf{R}$ being the $L \times L$ polarimetric covariance matrix.

Note that the single-channel AR model has been widely adopted in radar signal processing. For instance, different authors demonstrated that it can be used to reasonably approximate the spectral characteristics of different types of clutter [58]-[62], e.g. sea, ground, atmospheric. In this work, we extend this model by also considering the existing correlation between the available polarimetric channels. The model in (9) will be used in this paper for the theoretical derivation of an appropriate detector. Then, its suitability in practical applications will be verified in Part II of this two-part paper against both simulated and experimental data.

Based on this model, the approximate (actually conditional) probability density function (pdf) of the data [43][44][63][64] under the $H_{0}$ hypothesis can be written as:

$$
\begin{aligned}
& f_{0}\left(\mathbf{x}_{0} \mid \mathbf{R},\{\mathbf{A}(q)\}_{q=1}^{Q-1}\right)=\left(\pi^{L}|\mathbf{R}|\right)^{-(M-Q+1)} \times \\
& \exp \left\{-\sum_{m=Q}^{M}\left[\mathbf{x}_{0}(m)-\sum_{q=1}^{Q-1} \mathbf{A}^{H}(q) \mathbf{x}_{0}(m-q)\right]^{H}\right. \\
& \left.\times \mathbf{R}^{-1}\left[\mathbf{x}_{0}(m)-\sum_{q=1}^{Q-1} \mathbf{A}^{H}(q) \mathbf{x}_{0}(m-q)\right]\right\}
\end{aligned}
$$

being $\mathbf{x}_{0}$ defined in (3).

We note that the conditional pdf in (10) well approximates the actual pdf of the data for the large-sample case [63][64]. Therefore, as in [51][52], we use (10) in the following to derive the sought detector and to perform the ML estimation of the AR parameters. A similar expression can be provided under $H_{1}$, by replacing each instance of $\mathbf{x}_{0}(m)$ with $\mathbf{x}_{0}(m)-$ $\mathbf{S}(m)$.

To simplify the notation, we arrange the matrix parameters of the $\operatorname{AR}(Q-1)$ process in a $L(Q-1) \times L$ matrix $\mathbf{A}=\left[\mathbf{A}^{H}(Q-\right.$ 1) $\left.\mathbf{A}^{H}(Q-2) \ldots \mathbf{A}^{H}(1)\right]^{H}$. In addition, we define the vectors $\tilde{\mathbf{x}}_{0}(m)=\left[\mathbf{x}_{0}^{H}(m) \mathbf{x}_{0}^{H}(m+1) \ldots \mathbf{x}_{0}^{H}(m+Q-1)\right]^{H}, m=0$, $\ldots, M-Q$, which collect $Q$ consecutive snapshots of the data starting from the $m$-th sample.

Finally, we arrange these vectors into matrix $\mathbf{X}_{0}(L Q \times L(M$ $-Q+1))$ :

$$
\mathbf{X}_{0}=\left[\begin{array}{llll}
\tilde{\mathbf{x}}_{0}(0) & \tilde{\mathbf{x}}_{0}(1) & \ldots & \tilde{\mathbf{x}}_{0}(M-Q)
\end{array}\right]
$$

The same definitions are extended to the target components, yielding the following matrix structures:

$$
\begin{gathered}
\mathbf{S}=\left[\begin{array}{lll}
\tilde{\mathbf{s}}(0) & \widetilde{\mathbf{s}}(1) \ldots \tilde{\mathbf{s}}(M-Q)
\end{array}\right] \\
\widetilde{\mathbf{s}}(m)=\left[\begin{array}{l}
\mathbf{s}^{H}(m) \mathbf{s}^{H}(m+1) \ldots \mathbf{s}^{H}(m+Q-1)
\end{array}\right]^{H} \\
=\tilde{\mathbf{t}}(m) \otimes \boldsymbol{\alpha}
\end{gathered}
$$

where $\tilde{\mathbf{t}}(m)$ collects a $Q$-dimensional sub-vector of the temporal steering vector starting from the $m$-th sample.

Consequently, the likelihood of the data under hypothesis $H_{\gamma}(\gamma=0,1)$ can be rewritten as:

$$
\begin{aligned}
& f_{\gamma}\left(\mathbf{X}_{0} \mid \gamma \boldsymbol{\alpha}, \mathbf{R}, \mathbf{A}\right)=\left(\pi^{L}|\mathbf{R}|\right)^{-(M-Q+1)} \\
& \times \exp \left\{-\operatorname{tr}\left[\left(\mathbf{X}_{0}-\gamma \mathbf{S}\right)^{H} \mathbf{P}\left(\mathbf{X}_{0}-\gamma \mathbf{S}\right)\right]\right\}
\end{aligned}
$$

where $\mathbf{P}=\left[\mathbf{H}^{H} \mathbf{R}^{-1} \mathbf{H}\right], \mathbf{H}=\left[\begin{array}{ll}-\mathbf{A}^{H} & \mathbf{I}_{L}\end{array}\right]$, and $\operatorname{tr}(\mathbf{M})$ denotes the trace of matrix $\mathbf{M}$.

\section{B. AR model based Polarimetric Matched Filter}

We derive the polarimetric adaptive detector by resorting to a two-step GLRT design criterion. Specifically, in this section we assume that the parameters of the $\operatorname{AR}(Q-1)$ model, say $\mathbf{R}$ and A, are known, and we derive the GLRT detection test based on primary data. Then, in the next sub- 
section, a fully adaptive detector is obtained by replacing the unknown matrices with their ML estimates.

The test statistic of the GLRT based solely on primary data is given by:

$$
\frac{\max _{\boldsymbol{\alpha}}\left\{f_{1}\left(\mathbf{X}_{0} \mid \boldsymbol{\alpha}, \mathbf{R}, \mathbf{A}\right)\right\}}{f_{0}\left(\mathbf{X}_{0} \mid \mathbf{R}, \mathbf{A}\right)} \underset{H_{0}}{\stackrel{H_{1}}{\gtrless} \eta_{0}}
$$

where $\eta_{0}$ is the detection threshold.

By maximizing the numerator over $\boldsymbol{\alpha}$ and after some algebraic manipulations (see Appendix A), we obtain the following test statistic:

$$
\begin{aligned}
& T_{P o l-A R-M F} \\
& =\sum_{m=0}^{M-Q} \tilde{\mathbf{x}}_{0}^{H}(m) \mathbf{P} \boldsymbol{\Sigma}(m)\left[\frac{1}{2} \sum_{m=0}^{M-Q} \boldsymbol{\Sigma}^{H}(m) \mathbf{P} \boldsymbol{\Sigma}(m)\right]^{-1} \\
& \times \sum_{m=0}^{M-Q} \boldsymbol{\Sigma}^{H}(m) \mathbf{P} \tilde{\mathbf{x}}_{0}(m) \underset{H_{0}}{\stackrel{H_{1}}{\gtrless} \eta_{A R-M F}}
\end{aligned}
$$

where $\boldsymbol{\Sigma}(m)=\tilde{\mathbf{t}}(m) \otimes \mathbf{I}_{L}$. The detection scheme in (16) will be referred to in the following as the polarimetric AR model based matched filter (Pol-AR-MF).

The test statistic above can be interpreted by defining the matrices $\mathbf{W}=\frac{1}{2} \sum_{k=0}^{M-Q} \boldsymbol{\Sigma}^{H}(m) \mathbf{P} \boldsymbol{\Sigma}(m)$ and $\mathbf{V}(m)=\mathbf{P} \boldsymbol{\Sigma}(m)$, $m=0, \ldots, M-Q$, which allows us to rewrite (16) as:

$$
\begin{gathered}
T_{P o l-A R-M F}=\sum_{m=0}^{M-Q} \tilde{\mathbf{x}}_{0}^{H}(m) \mathbf{V}(m) \mathbf{W}^{-1} \\
\times \sum_{m=0}^{M-Q} \mathbf{V}^{H}(m) \tilde{\mathbf{x}}_{0}(m) \underset{H_{1}}{\stackrel{H_{1}}{\gtrless} \eta_{A R-M F}}
\end{gathered}
$$

We observe that $\mathbf{V}(m)(L Q \times L)$ includes the filter coefficients to be applied at the $k$-th sub-coherent processing interval (CPI) to obtain the temporally whitened sequence of $L$-dimensional vectors $\mathbf{y}_{0}(m)=\mathbf{V}^{H}(m) \tilde{\mathbf{x}}_{0}(m), m=0, \ldots, M$ $-Q$. The summation across consecutive samples provides the coherent integration of target echoes in time domain, which yields $\mathbf{z}_{0}=\sum_{m=0}^{M-Q} \mathbf{y}_{0}(m)$. Notice that, assuming a block based implementation of the detection scheme, the exploitation of an order $Q-1$ for the AR model implies a loss of $Q-1$ samples on the sequence $\mathbf{y}_{0}(\mathrm{~m})$, namely the summation is limited to $M$ $-Q+1$ samples. This might be responsible of limited loss as observed in the results reported in Section V. However, other implementations are possible based on lattice filters operating across the slow time.

Finally, the test statistic is evaluated as $T_{P o l-A R-M F}=$ $\mathbf{z}_{0}^{H} \mathbf{W}^{-1} \mathbf{z}_{0}$ that encodes the polarimetric whitening followed by the non-coherent integration of target echoes across the polarimetric channels. This is a direct consequence of the partially unstructured model adopted for the target component (see (4) and subsequent positions).

Notice that, if $\mathbf{t}$ represents the temporal steering vector, namely the vector that encodes the target echo phase shifts across consecutive radar pulses, we can rework and simplify the expression in (17). To this purpose, we observe that $\tilde{\mathbf{t}}(m)=e^{j 2 \pi f_{d}^{m}} \tilde{\mathbf{t}}(0)$ and, consequently, we can write:

$$
\begin{gathered}
T_{\text {Pol-AR-MF }}=\sum_{m=0}^{M-Q} e^{j 2 \pi f_{d} m} \tilde{\mathbf{x}}_{0}^{H}(m) \mathbf{V}(0) \mathbf{W}^{-1} \\
\times \sum_{m=0}^{M-Q} e^{-j 2 \pi f_{d} m} \mathbf{V}^{H}(0) \tilde{\mathbf{x}}_{0}(m) \stackrel{H_{1}}{\underset{H_{0}}{\gtrless} \eta_{A R-M F}}
\end{gathered}
$$

where $\mathbf{W}=\frac{1}{2}(M-Q+1) \boldsymbol{\Sigma}^{H}(0) \mathbf{P} \boldsymbol{\Sigma}(0)$.

Therefore, in this case, a constant filter can be applied to obtain the whitened sequence $\mathbf{y}_{0}^{\prime}(m)=\mathbf{V}^{H}(0) \tilde{\mathbf{x}}_{0}(m)$, provided that the phase shift across consecutive sub-CPIs is compensated for before coherent integration. The above simplification also allows an alternative implementation of the temporal whitening stage as the filtering by matrix $\mathbf{V}(0)$ and the summation could be performed in reverse order to limit the computational burden.

As a last remark, we notice that the test statistic in (17) can be expressed as a quadratic form in $\mathbf{x}_{0}$ as:

$$
T_{\text {Pol-AR-MF }}=\mathbf{x}_{0}^{H} \mathbf{B} \mathbf{C} \mathbf{C}^{H} \mathbf{B}^{H} \mathbf{x}_{0} \underset{1}{H_{1}} \eta_{A R-M F}
$$

where

$$
\begin{aligned}
& \boldsymbol{B}=\left[\begin{array}{llll}
\mathbf{B}_{0} & \mathbf{B}_{1} & \ldots & \mathbf{B}_{M-Q}
\end{array}\right] \\
& \text { with } \mathbf{B}_{m}=\left[\begin{array}{c}
\mathbf{0}_{L m \times L} \\
\mathbf{V}(m) \\
\mathbf{0}_{L(M-Q-m) \times L}
\end{array}\right] m=0, \ldots, M-Q \\
& \mathbf{C}=\left(\mathbf{1}_{M-Q+1 \times 1} \otimes \mathbf{W}^{-\frac{1}{2}}\right)
\end{aligned}
$$

Under the assumptions adopted in (18), the blocks of matrix $\mathbf{B}$ are shifted versions of the first block, being the shift by $L$ elements row-wise, whereas $\mathbf{C}=\left(\overline{\mathbf{t}} \otimes \mathbf{W}^{-\frac{1}{2}}\right)$ where $\overline{\mathbf{t}}$ includes the first $M-Q+1$ elements of the steering vector $\mathbf{t}$.

\section{Adaptive implementation}

To make the derived detector fully adaptive, matrix $\mathbf{P}$, and hence matrices $\mathbf{A}$ and $\mathbf{R}$, must be replaced with their ML estimates. These are obtained from the secondary data $\mathbf{x}_{p}, p=$ $1, \ldots, P$, for which the same assumptions adopted in Section II hold (i.i.d. and target free). Specifically, in this case we assume that the disturbance in the secondary data follows an $\operatorname{AR}(Q-1)$ model with same parameters of the disturbance affecting the primary data.

By applying the same reordering strategy as for $\mathbf{X}_{0}$ (see (11)) to the secondary data vectors, we obtain $P$ matrices $\mathbf{X}_{p}$, $p=1, \ldots, P$, that are then collected in a larger matrix $\overline{\mathbf{X}}=$ $\left[\mathbf{X}_{1} \ldots \mathbf{X}_{P}\right]$ of dimensions $Q L \times P(M-Q+1)$.

The joint likelihood of the secondary data is then written as: 


$$
\begin{aligned}
& f_{0}(\overline{\mathbf{X}} \mid \mathbf{R}, \mathbf{A}) \\
& =\left(\pi^{L}|\mathbf{R}|\right)^{-P(M-Q+1)} \exp \left\{-\operatorname{tr}\left(\overline{\mathbf{X}}^{H} \mathbf{P} \overline{\mathbf{X}}\right)\right\}
\end{aligned}
$$

Based on (22), proper approximation of the ML estimates of $\mathbf{A}$ and $\mathbf{R}$ are readily obtained as (see Appendix B):

$$
\widehat{\mathbf{A}}=\widehat{\mathbf{Q}}_{00}^{-1} \widehat{\mathbf{Q}}_{01}
$$

and

$$
\widehat{\mathbf{R}}=\frac{1}{P(M-Q+1)}\left(\widehat{\mathbf{Q}}_{11}-\widehat{\mathbf{Q}}_{01}^{H} \widehat{\mathbf{Q}}_{00}^{-1} \widehat{\mathbf{Q}}_{01}\right)
$$

where $\widehat{\mathbf{Q}}_{00}(L(Q-1) \times L(Q-1)), \widehat{\mathbf{Q}}_{01}(L(Q-1) \times L), \widehat{\mathbf{Q}}_{11}(L \times L)$ are blocks of the following matrix:

$$
\widehat{\mathbf{Q}}=\overline{\mathbf{X}} \overline{\mathbf{X}}^{H}=\left[\begin{array}{ll}
\widehat{\mathbf{Q}}_{00} & \widehat{\mathbf{Q}}_{01} \\
\widehat{\mathbf{Q}}_{01}^{H} & \widehat{\mathbf{Q}}_{11}
\end{array}\right]
$$

Notice that a similar result was obtained in [51]-[52],[54] since this is independent of the adopted model for the target components.

We observe that the $L Q \times L Q$ matrix $\widehat{\mathbf{Q}}$ represents an estimate of the disturbance covariance matrix within a subCPI, namely any $L Q \times L Q$ block on the main diagonal of matrix M. This benefits from a joint average over secondary data and consecutive (overlapped) sub-CPIs within the CPI:

$$
\widehat{\mathbf{Q}}=\sum_{p=1}^{P} \sum_{m=0}^{M-Q} \tilde{\mathbf{x}}_{p}(m) \tilde{\mathbf{x}}_{p}^{H}(m)
$$

The need for a smaller number of parameters to be estimated and the possibility to improve the estimation stage based on consecutive temporal observations formed within the CPI allow to limit the adaptivity loss compared to the detection schemes in Section II [4]-[6].

By using (23) and (24) in (16), we obtain the test statistic for the polarimetric AR model based adaptive matched filter (Pol-AR-AMF) as

$$
\begin{aligned}
& T_{P o l-A R-A M F} \\
& =\sum_{m=0}^{M-Q} \tilde{\mathbf{x}}_{0}^{H}(m) \widehat{\mathbf{P}} \boldsymbol{\Sigma}(m)\left[\frac{1}{2} \sum_{m=0}^{M-Q} \boldsymbol{\Sigma}^{H}(m) \widehat{\mathbf{P}} \boldsymbol{\Sigma}(m)\right]^{-1} \\
& \times \sum_{m=0}^{M-Q} \boldsymbol{\Sigma}^{H}(m) \widehat{\mathbf{P}} \tilde{\mathbf{x}}_{0}(m) \stackrel{H_{1}}{\gtrless} \eta_{A R-A M F} \\
& H_{0}
\end{aligned}
$$

being $\widehat{\mathbf{P}}=\left[\widehat{\mathbf{H}}^{H} \widehat{\mathbf{R}}^{-1} \widehat{\mathbf{H}}\right]$ and $\widehat{\mathbf{H}}=\left[\begin{array}{ll}-\widehat{\mathbf{A}}^{H} & \mathbf{I}_{L}\end{array}\right]$. Similarly, the adaptive versions of the test statistics in (18)-(19) can be easily obtained.

\section{Asymptotic PeRformance}

In this Section, by proceeding as in [52], we derive the asymptotic performance achievable with the proposed detector, i.e. under the assumption that $\widehat{\mathbf{A}}$ and $\widehat{\mathbf{R}}$ are asymptotic estimates obtained from an infinite number of secondary data [64]. Basically, we neglect the adaptivity loss due to the estimate fluctuations and, provided that the pdf in eq.(10) correctly approximates the actual pdf of the data for the large-sample case, we assume that the asymptotic ML estimates of $\mathbf{A}$ and $\mathbf{R}$ coincide with the actual values of the AR parameters. This analysis is useful since it might be representative of the performance of its adaptive version for large number of training data.

\section{A. False alarm probability}

Let us consider the test statistic in (19) and define the $L_{-}$ dimensional vector $\breve{\mathbf{z}}_{0}=\mathbf{C}^{H} \mathbf{B}^{H} \mathbf{x}_{0}$, namely $\breve{\mathbf{z}}_{0}$ represents the data after both polarimetric and temporal filtering, which then undergoes the non-coherent integration across the polarimetric channels and the test statistic in (19) can be written as $T_{P o l-A R-M F}=\left\|\breve{\mathbf{z}}_{0}\right\|^{2}$.

If the input disturbance process exactly matches the $\operatorname{AR}(Q$ - 1) model exploited for the derivation of the proposed detector, the filtering of data $\mathbf{x}_{0}$ via matrices $\mathbf{B}$ and $\mathbf{C}$, based on asymptotic estimates of the relevant parameters included therein, provides a perfect whitening in both the polarimetric and the temporal domain. Under the $H_{0}$ hypothesis, $\breve{\mathbf{z}}_{0}$ is a complex Gaussian random vector with zero-mean and covariance matrix $2 \mathbf{I}_{L}$, i.e. $\left.\breve{\mathbf{z}}_{0}\right|_{H_{0}} \sim \mathcal{C N}\left(\mathbf{0}_{L \times 1}, 2 \mathbf{I}_{L}\right)$. See Appendix $\mathrm{C}$ for demonstration.

Thus, the asymptotic distribution of the test statistic of the adaptive detector is given by

$$
T_{\text {Pol-AR-AMF }} \stackrel{\text { asymp. }}{\longrightarrow} T_{P o l-A R-M F} \sim \chi_{2 L}^{2}(0)
$$

where $\chi_{2 L}^{2}(0)$ denotes the central Chi-squared distribution with $2 L$ degrees of freedom. Correspondingly, we can write the asymptotic false alarm probability $P_{f a}$ as

$$
P_{f a}=\sum_{l=0}^{L-1} \frac{\eta^{l}}{2^{l} \Gamma(L-l)} e^{-\frac{\eta}{2}}
$$

where $\Gamma(\cdot)$ is the Gamma function and $\eta$ is the threshold that guarantees the desired $P_{f a}$. Eq. (29) gives an exact threshold $\eta_{A R-M F}$ for the clairvoyant detector in (19) whereas, as the number $P$ of secondary data increases, it provides a good approximation of the threshold $\eta_{A R-A M F}$ to be adopted for the adaptive detector in (27).

The asymptotic distribution of $T_{P O L-A R-A M F}$ under $H_{0}$ is independent of the unknown parameters. Consequently, the $P_{f a}$ in (29) depends only on the test threshold and the number of polarimetric channels, and these are design parameters. It is then evident that the proposed detector asymptotically exhibits the CFAR property. 


\section{B. Probability of detection}

\section{1) Non-fluctuating target model (Swerling 0)}

For a non-fluctuating target model, namely a Swerling 0 target [65], and assuming know the disturbance parameters, vector $\breve{\mathbf{z}}_{0}$ under hypothesis $H_{1}$ is a complex Gaussian random vector, with mean vector $\mathbf{v}=\mathbf{C}^{H} \mathbf{B}^{H} \mathbf{s}$ and covariance matrix $2 \mathbf{I}_{L}$, i.e. $\left.\breve{\mathbf{z}}_{0}\right|_{H_{1}} \sim \mathcal{C N} \mathcal{N}\left(\mathbf{v}, 2 \mathbf{I}_{L}\right)$, and the asymptotic distribution of the test statistic is given by

$$
T_{\text {Pol-AR-AMF }} \stackrel{\text { asymp. }}{\longrightarrow} T_{P o l-A R-M F} \sim \chi_{2 L}^{2}(\mathrm{~s})
$$

where $\chi_{2 L}^{2}(\varsigma)$ denotes the noncentral Chi-squared distribution with $2 L$ degrees of freedom and noncentrality parameter $\varsigma=$ $\sum_{l=0}^{L-1}\left|v_{l}\right|^{2}=\|\mathbf{v}\|^{2}$.

From (30), we can write the asymptotic detection probability $P_{d}$ expression using the Marcum Q-function, as follows

$$
\begin{aligned}
& P_{d}=Q_{L}(\sqrt{\varsigma}, \sqrt{\eta}) \\
& =\int_{\sqrt{\eta}}^{\infty} x\left(\frac{x}{\sqrt{\varsigma}}\right)^{L-1} \exp \left(-\frac{x^{2}+\varsigma}{2}\right) I_{L-1}(\sqrt{\varsigma} x) d x
\end{aligned}
$$

$I_{L-1}(\sqrt{\varsigma} x)$ being the modified Bessel function of order $L-1$.

Eqs. (31) and (29) extend the results in [50] that were obtained for the case of a structured model for the space-time target components. That model basically yields a coherent summation over the whitened sequences and, in turn, results in a $\chi_{2}^{2}(\gamma \varsigma)$ asymptotic distribution for the test statistic under $\mathrm{H}_{\gamma}(\gamma=0,1)$.

\section{2) Fluctuating target model (Swerling I)}

Assuming a Swerling I model for the target [65], namely considering a target complex amplitude distributed as a zeromean Gaussian random variable with covariance matrix $\mathbf{M}_{t}=$ $E\left\{\boldsymbol{\alpha} \boldsymbol{\alpha}^{H}\right\}$, vector $\breve{\mathbf{z}}_{0}$ turns into a complex Gaussian random variable with zero-mean vector and covariance matrix $\mathbf{D}_{0}=$ $2 \mathbf{I}_{L}+\mathbf{C}^{H} \mathbf{B}^{H}\left(\mathbf{t t}^{H} \otimes \mathbf{M}_{\mathrm{t}}\right) \mathbf{B C}$.

Therefore, in this case, we look for a closed form expression for the asymptotic $P_{d} \triangleq \operatorname{Prob}\left\{\left\|\breve{\mathbf{z}}_{0}\right\|^{2}>\eta \mid H_{1}\right\}$, with $\left.\breve{\mathbf{z}}_{0}\right|_{H_{1}} \sim \mathcal{C N} \mathcal{N}\left(\mathbf{0}_{L \times 1}, \mathbf{D}_{0}\right)$. To this purpose we can use some recent results from the theory of indefinite quadratic forms in Gaussian random variables [67]. To this end, let $\lambda_{0} \ldots \lambda_{N-1}$ denote the $N \leq L$ distinct non-zero eigenvalues of $\mathbf{D}_{0}$, each with multiplicity $\mu_{n}, n=0, \ldots, N-1$. Following the approach in [67], we can write the $P_{d}$ expression as follows (see Appendix D for the derivation):

$$
P_{d}=\sum_{n=0}^{N-1} \sum_{k=0}^{\mu_{n}-1} \frac{-e^{-\frac{\eta}{\lambda_{n}}} \eta^{k}}{\Gamma(k+1)} \delta_{k, n}
$$

where $\eta$ is the threshold, and the coefficients $\delta_{k, n}$ definition is detailed in eq. (56) of Appendix D. The expression above takes simplified forms for the special cases of either a unique eigenvalue $\left(\lambda_{0}\right)$ with multiplicity $L$ or $L$ distinct eigenvalues. Specifically, in the former case we obtain

$$
P_{d}=\sum_{l=0}^{L-1} \frac{\eta^{l}}{\lambda_{0}^{l} \Gamma(l+1)} e^{-\frac{\eta}{\lambda_{0}}}
$$

which corresponds to a Gamma distributed test statistic, i.e.

$$
T_{\text {Pol-AR-AMF }} \stackrel{\text { asymp. }}{\longrightarrow} T_{\text {Pol-AR-MF }} \sim \Gamma\left(L, \lambda_{0}\right)
$$

whereas, in the case of $L$ distinct eigenvalues, we have

$$
P_{d}=\sum_{l=0}^{L-1} \frac{\lambda_{l}^{L-1}}{\prod_{\substack{i=0 \\ i \neq l}}^{L-1}\left(\lambda_{l}-\lambda_{i}\right)} \mathrm{e}^{-\frac{\eta}{\lambda_{l}}}
$$

which yields, for the test statistic, the following asymptotic probability density function (pdf):

$$
\begin{aligned}
& T_{\text {Pol-AR-AMF }} \stackrel{\text { asymp. }}{\longrightarrow} \\
& T_{P o l-A R-M F} \sim \sum_{l=0}^{L-1} \frac{\lambda_{l}^{L-2}}{\prod_{\substack{i=0 \\
i \neq l}}^{L-1}\left(\lambda_{l}-\lambda_{i}\right)} \mathrm{e}^{-\frac{\eta}{\lambda_{l}}}
\end{aligned}
$$

\section{NUMERICAL RESULTS}

In this Section, we investigate the performance of the proposed detector via numerical examples.

To this aim, we generate the disturbance signal as a $L-$ channel $\operatorname{AR}(Q-1)$ process and matrices $\mathbf{A}$ and $\mathbf{R}$ and we carry out Monte Carlo (MC) simulations with proper number of trials. In particular, we consider an order $Q-1=3$ for the AR process with $L=3$ ( $\mathrm{HH}, \mathrm{VV}, \mathrm{HV}$ ) polarimetric channels.

The $\operatorname{AR}(Q-1)$ parameters $\mathbf{A}$ and $\mathbf{R}$ are set so that the autoand cross- spectra of the available channels are those reported in Fig. 1 [68]. Specifically, Fig. 1 shows that, in the considered example, the disturbance at channels $\mathrm{HH}$ and VV has identical spectral characteristics and equal power level, i.e. $\sigma_{d, H H}^{2}=$ $\sigma_{d, V V}^{2}=\sigma_{d}^{2}$, deliberately set to $\sigma_{d}^{2}=1$.

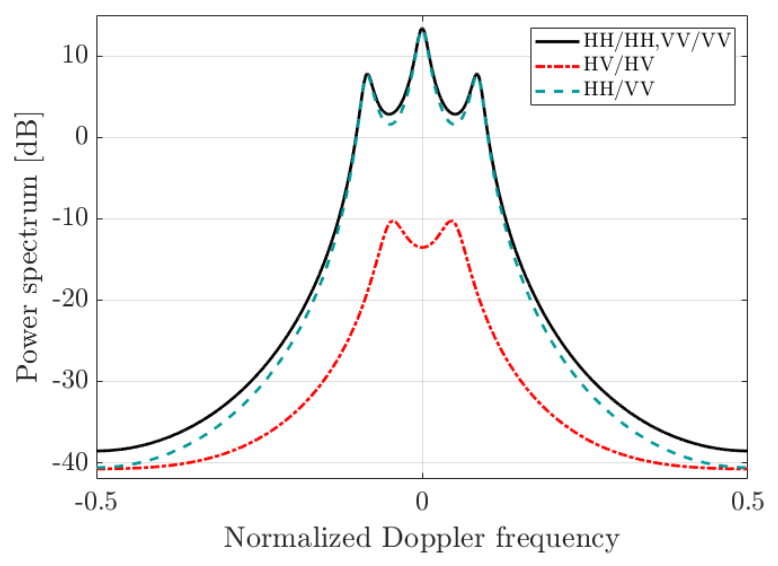

Fig. 1 Power spectra of a $L$-channel AR(3) process 
The cross-spectrum between the $\mathrm{HH}$ and VV channels reveals a good correlation between the corresponding signals yielding a correlation coefficient equal to $\rho_{H H / V V}=0.9$. The disturbance affecting the $\mathrm{HV}$ channel is generated with power level $20 \mathrm{~dB}$ lower than in $\mathrm{HH}$ and VV, i.e. $\sigma_{d, H V}^{2}=0.01 \sigma_{d}^{2}$. Moreover, the cross-polarized components are assumed independent from the co-polarized ones, i.e. $\rho_{H V / H H}=$ $\rho_{H V / V V}=0$ thus resulting in null cross-spectra.

In Fig. 2 we study the capability of the derived expression to control the false alarm rate as a function of the number of training data. Specifically, for a given desired $P_{f a}=10^{-3}$, we select the threshold according to the asymptotic expression in (29). Then, we perform MC simulations with $10^{6}$ trials and different $P$ values, and we evaluate the actual $P_{f a}$ obtained when using the theoretical threshold.

The results are reported for different numbers $L$ of polarimetric channels in Fig. 2 (a) whereas in Fig. 2 (b) we compare the curves obtained for different numbers $M$ of temporal observations as provided by a coherent train of pulses (an active pulse radar system is assumed in the reported examples).

By observing Fig. 2 the following considerations are in order.

- In all considered cases, the actual $P_{f a}$ tends to the nominal false alarm used to set the threshold $\left(P_{f a}=10^{-3}\right)$ as the number $P$ of secondary data increases. This on one hand confirms the correctness of the asymptotic expression in (29) and on the other hand demonstrates that the detection threshold obtained from (29) could be exploited in practice when the number of secondary data is sufficiently high. When this condition does not hold, adaptivity loss prevails, which yields a false alarm rate higher than the desired one.

- By keeping the number of pulses constant $(M=32$ in Fig. 2 (a)), the higher the number of polarimetric channels is the higher is the number of training data required to have acceptable adaptivity loss. This is because a bigger $(Q L$ $\times Q L)$ matrix must be estimated, being $Q=4$ in the considered case study.

- However, we observe that, in this case, a number $P$ of training data equal to $Q L$ is typically enough to guarantee the 'asymptotic' condition since the matrix estimation benefits from the average performed across the temporal observations within the CPI.

- The consideration above is confirmed in Fig. 2 (b) where we kept the number of polarimetric channels constant $(L=$ 2 ), while comparing the results obtained for different values of $M$. In fact, as the number of pulses increases, the training data required to have a good false alarm rate control decrease. This is because a higher number of consecutive temporal observations is available to estimate the same number of unknown parameters.

The detection performance of the proposed detector is analyzed in Fig. 3 and Fig. 4, by comparison with the PolGLRT [4]-[5] in (8) and the Pol-AMF [6] in (7).

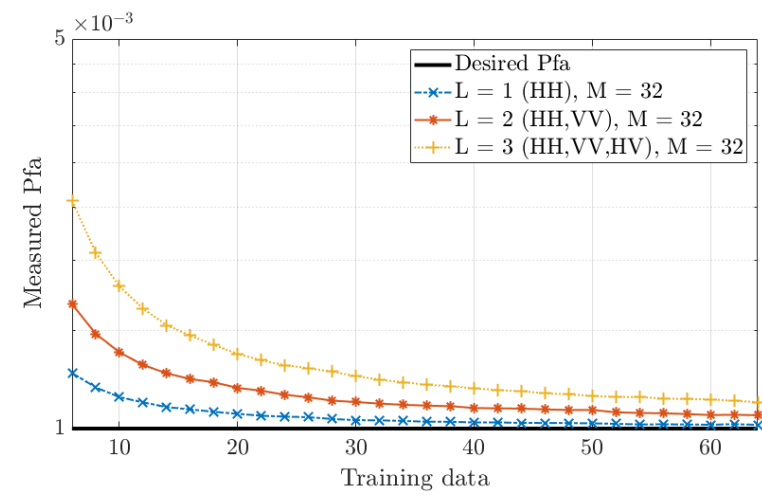

(a)

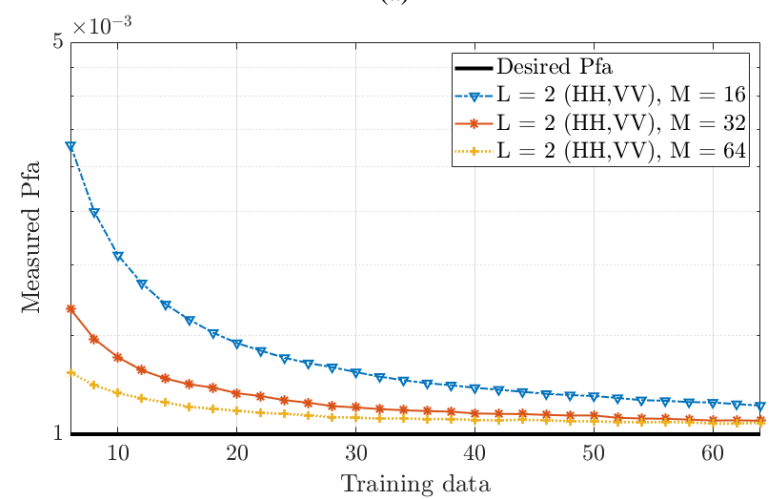

(b)

Fig. 2 Measured $P_{f a}$ versus $P$ when $P_{f a}=10^{-3}$ for

(a) different polarimetric channels and (b) different pulses

First, a Swerling 0 target model is considered in Fig. 3, namely the target complex amplitudes are assumed deterministic [65]. As an example, in the considered case study, they are set as $\boldsymbol{\alpha}=a_{t}\left[\begin{array}{lll}1 & \mathrm{e}^{j \Delta \phi_{H H / V V}} & \sqrt{\xi_{t}} \mathrm{e}^{j \Delta \phi_{H H / H V}}\end{array}\right]^{T}$, where $\xi_{t}=0.1$, $\Delta \phi_{H H / V V}=\pi / 4$, and $\Delta \phi_{H H / H V}=\pi / 2$. The results are reported as a function of the signal-to-clutter ratio (SCR) at the first polarimetric channel, i.e. SCR $=\left|a_{t}\right|^{2} / \sigma_{d}^{2}$.

We consider $M=32$, target normalized Doppler frequency $f_{d}=0.25$ and different numbers of polarimetric channels. Specifically, in Fig. 3(a) we use $L=1$ (HH) while in Fig. 3(b) we consider $L=3$ ( $\mathrm{HH}, \mathrm{VV}, \mathrm{HV})$. We note that, when $L=1$, the proposed detector falls within the single-channel parametric approaches [41]-[46] that only exploit the temporal domain for clutter cancellation and target detection.

Then, the same case studies are considered in Fig. 4 except that a fluctuating target model is used according to a Swerling I model [65]. Note that, in both cases, the employed parameters yield a ratio between the powers on the different polarimetric channels similar to that used in [5]. Specifically, the target complex amplitudes vector $\boldsymbol{\alpha}$ is generated as a zeromean Gaussian random vector, with the following covariance matrix

$$
\mathbf{M}_{t}=\sigma_{t}^{2}\left[\begin{array}{ccc}
1 & \rho_{t} & 0 \\
\rho_{t} & 1 & 0 \\
0 & 0 & \xi_{t}
\end{array}\right]
$$




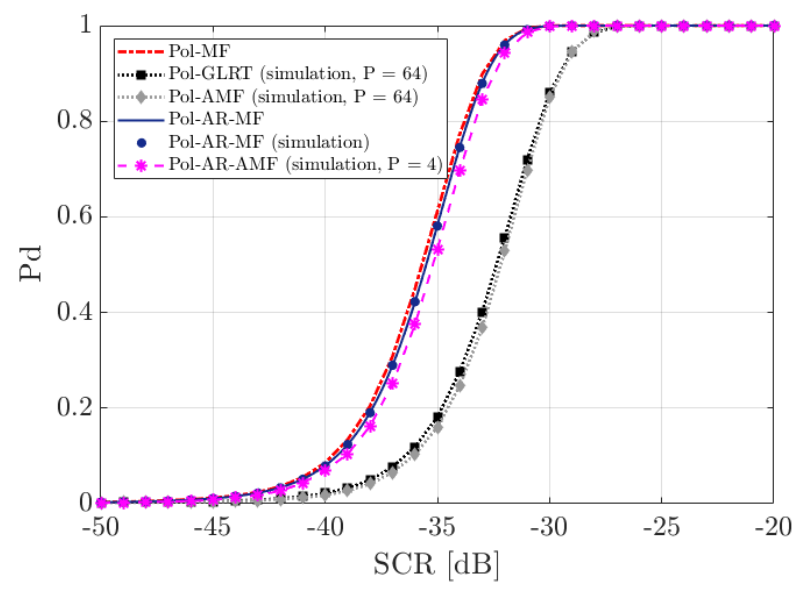

(a)

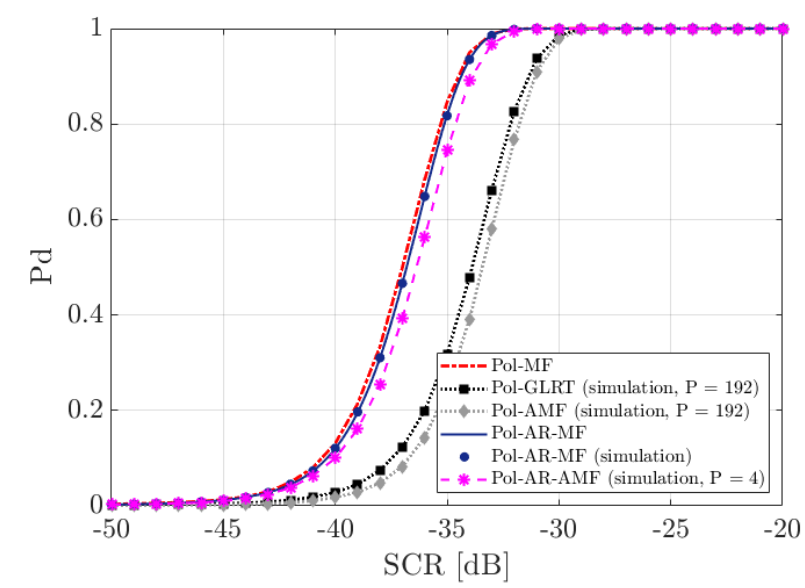

(b)

Fig. $3 P_{d}$ versus SCR for $M=32, P_{f a}=10^{-3}$, Swerling 0 target model and different polarimetric channels:

(a) $L=1(\mathrm{HH})$, (b) $L=3(\mathrm{HH}, \mathrm{VV}, \mathrm{HV})$

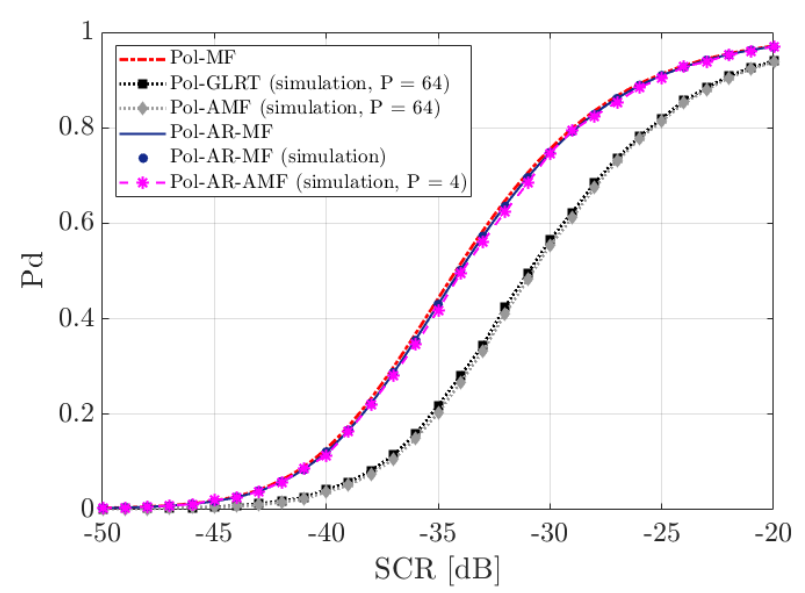

(a)

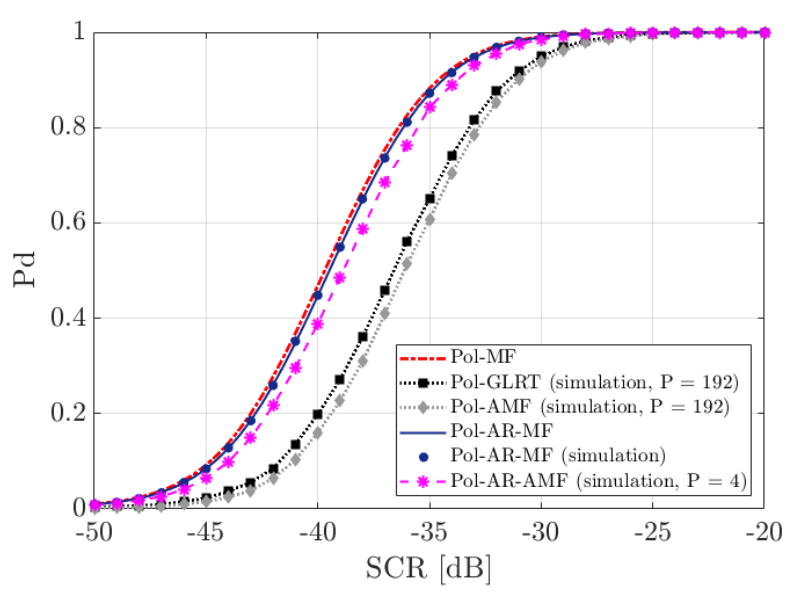

(b)

Fig. $4 P_{d}$ versus SCR for $M=32, P_{f a}=10^{-3}$,Swerling I target model and different polarimetric channels: (a) $L=1(\mathrm{HH})$, (b) $L=3(\mathrm{HH}, \mathrm{VV}, \mathrm{HV})$

denoting $\rho_{t}$ as the cross-correlation coefficient between the two co-polarized target amplitudes. In this analysis, $\rho_{t}=0$ and $\xi_{t}=0.1$. Again, Fig. 4(a) and Fig. 4(b) have been obtained for $L=1(\mathrm{HH})$ and $L=3(\mathrm{HH}, \mathrm{VV}, \mathrm{HV})$ polarimetric channels, respectively. In all figures, the Pol-MF is considered as a benchmark of our performance evaluation and its performance are reported in dash-dot red. The performance of the PolGLRT and the Pol-AMF are reported in dotted black and grey, respectively, and both are operated using $P=2 M L$.

The theoretical asymptotic $P_{d}$ for the proposed detector is reported in continuous dark blue line while the results of the MC simulation for the clairvoyant Pol-AR-MF (with $10^{4}$ independent MC trials) are reported in dark blue dots. The MC simulation results obtained when applying the adaptive detector Pol-AR-AMF, with $P$ secondary data are reported in dashed magenta.

The $P_{f a}$ has been chosen to be $10^{-3}$ and, for a fair comparison, the detection threshold of the Pol-AR-AMF has been numerically adjusted to guarantee the desired false alarm rate even in non-asymptotic regime.

By observing Fig. 3 and Fig. 4, the following considerations apply.

- The Pol-MF sets the performance bounds, that improve moving from a system equipped with single polarimetric channel to a fully polarimetric system employing $L=3$ channels, thanks to the enhanced capability to discriminate target echoes from disturbance. The improvement is larger for the Swerling I target model since the polarimetric channels combination also allows the amplitude fluctuations to be averaged out thus removing some of the target fades.

- The Pol-GLRT and the Pol-AMF yield a non-negligible detection loss (3-4 dB) with respect to their clairvoyant version (Pol-MF) employing all the available degrees of freedom for disturbance removal. This is due to the need to estimate a very high number of unknown parameters when 
no model is adopted for the disturbance spectral characteristics. As expected, the Pol-GLRT shows a slight advantage over the Pol-AMF. However, the number of secondary data for both detectors has been adjusted so that $P=2 M L$, which yields $P=64$ and $P=192$ for $L=1$ and $L$ $=3$, respectively. In practical cases it may be difficult to obtain the required amount of training data with the desired characteristics so that further degraded performance is expected for both the fully adaptive detection schemes.

- Whilst it exploits a reduced number of temporal degrees of freedom for clutter cancellation, the Pol-AR-MF shows comparable performance with respect to the Pol-MF provided that the number $Q$ of taps matches the actual order of the AR process modelling the disturbance. The case of a possible mismatch between the disturbance spectral characteristics and the order of the AR process used to build the detector is addresses in the accompanying paper [55].

- The slight loss observed in Fig. 3 and Fig. 4 is due to the border effect arising from a block-based implementation of the detection scheme as discussed in Section III-B. In fact, according to this implementation, the summation across the sequence $\boldsymbol{y}_{0}(m)$ is limited to $M-Q-1$ consecutive samples thus a loss of $(Q-1) / M$ is obtained for the corresponding integration gain over the available CPI. Incidentally, we recall that other implementations are possible, e.g. based on lattice filters, where this loss might be avoided by processing partially overlapped data batches.

- The theoretical asymptotic $P_{d}$ expressions perfectly match with the MC simulation results, implying that the obtained expressions can accurately describe the detection performance of the Pol-AR-MF both under the deterministic and stochastic target models.

- The Pol-AR-AMF yields remarkable detection performance with only a limited adaptivity loss with respect to its clairvoyant version Pol-AR-MF. As expected, this loss increases as $L$ increases, since a bigger $(Q L \times Q L)$ matrix must be estimated and the number of training data has been kept constant ( $P=4$ in all considered cases).

- Nevertheless, the Pol-AR-AMF outperforms the other polarimetric adaptive detectors, even using much fewer training data. This advantage clearly demonstrates its suitability for practical applications.

In Fig. 5(a-c), we show the probability of detection for a Swerling I target model and $L=2$ polarimetric channels $(\mathrm{HH}$, $\mathrm{VV}$ ), when using a number $M$ of pulses equal to 16,32 , and 64, respectively. In all cases, the Pol-AR-AMF is applied with $P=4$ training data while both the Pol-GLRT and the Pol-AMF use $P=2 M L$, namely $P=64,128$ and 256. Similar considerations apply as for the results reported in Fig. 3 and Fig. 4.

In addition, by comparing Fig. 5(a-c), we further notice that, as the number $M$ of pulses increases:

- all target detection strategies benefit from the increased number of temporal observations that at least provides an increased coherent integration gain.

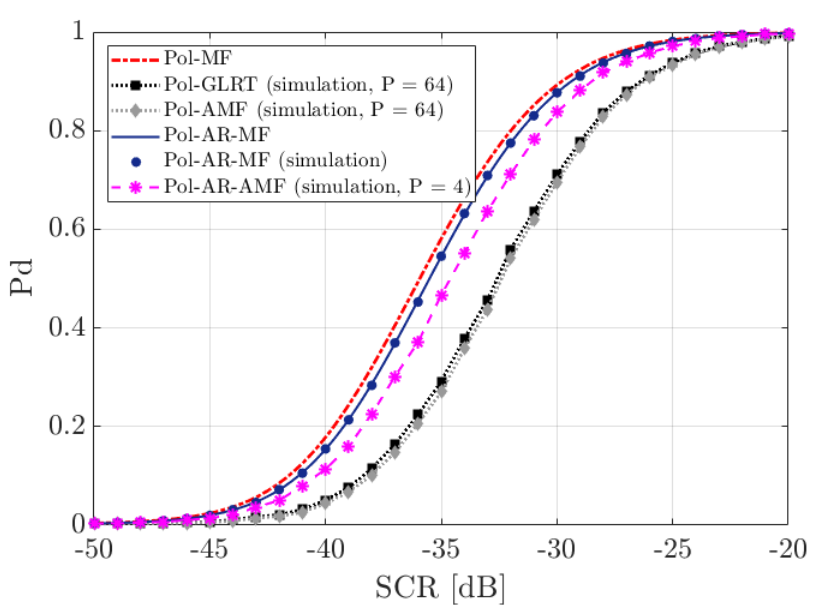

(a)

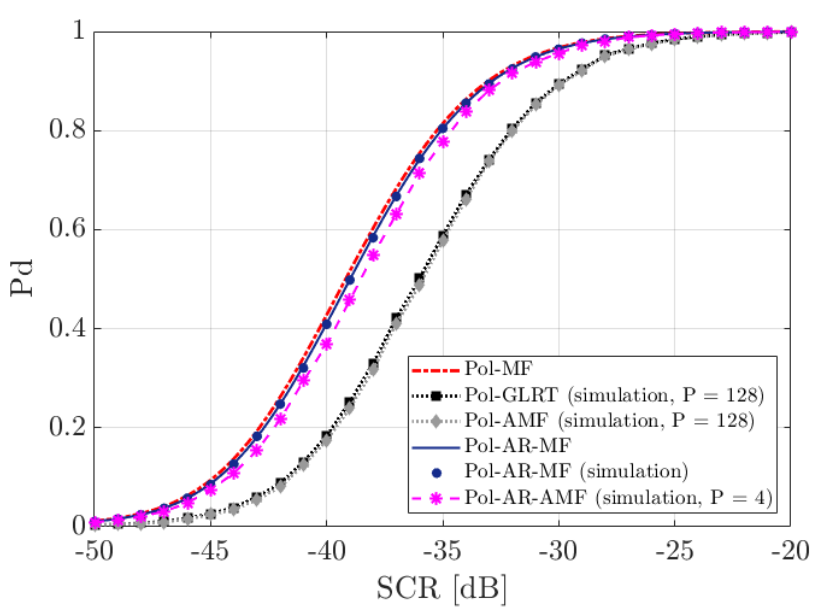

(b)

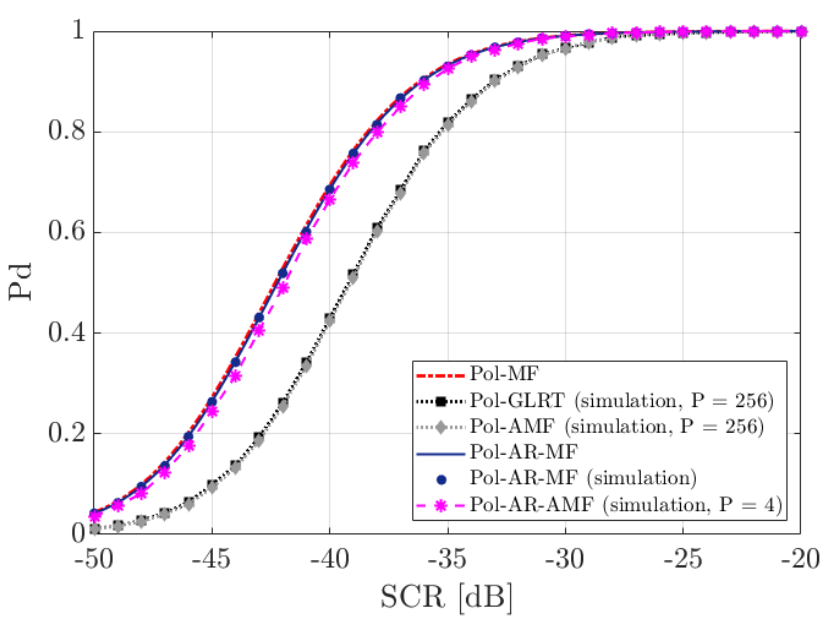

(c)

Fig. $5 P_{d}$ versus SCR for $P_{f a}=10^{-3}, L=2(\mathrm{HH}, \mathrm{VV})$ and different pulses: (a) $M=16$, (b) $M=32$, (c) $M=64$ 
- Moreover, the observed loss of the Pol-AR-AMF with respect to the Pol-MF due to the border effect decreases since the number of samples excluded from the coherent integration $(Q-1)$ becomes less significant with respect to $M$.

- To parity of training data, the adaptivity loss shown by the Pol-AR-AMF decreases thanks to the higher number of temporal observations available to estimate the same number of parameters $(Q L)$. In this regard, we observe that, even operating with $P=4$, in the considered case study, the Pol-AR-AMF substantially reaches its asymptotic detection performance for $M \geq 32$.

- In contrast, the conventional polarimetric adaptive detection schemes, i.e. the Pol-GLRT and the Pol-AMF, require a progressively higher number of secondary data in order to control the adaptivity loss, making such detection strategies unsuitable for real application. This conclusion is further reinforced by considerations relevant to the computational burdens required by these detectors.

- Overall, the advantage of the Pol-AR-AMF over the PolGLRT and the Pol-AMF increases as $M$ increases showing that the number of temporal degrees of freedom to be employed adaptively can be kept constant thus easing the training/computational burden while guaranteeing the desired disturbance removal. In fact, the Pol-AR-AMF allows the additional temporal observations to be effectively exploited to reduce the adaptivity loss and to enhance the coherent integration of target echoes.

Note that, although the devised comments specifically refer to the considered case study, similar considerations apply to alternative cases obtained with a different choice of the relevant parameters. For instance, we plot in Fig. 6 the results obtained when considering the same scenario used in Fig. 5(b) but assuming that the cross-correlation coefficient between the target amplitudes at channels $\mathrm{HH}$ and $\mathrm{VV}$ is equal to $\rho_{t}=$ 0.99 .

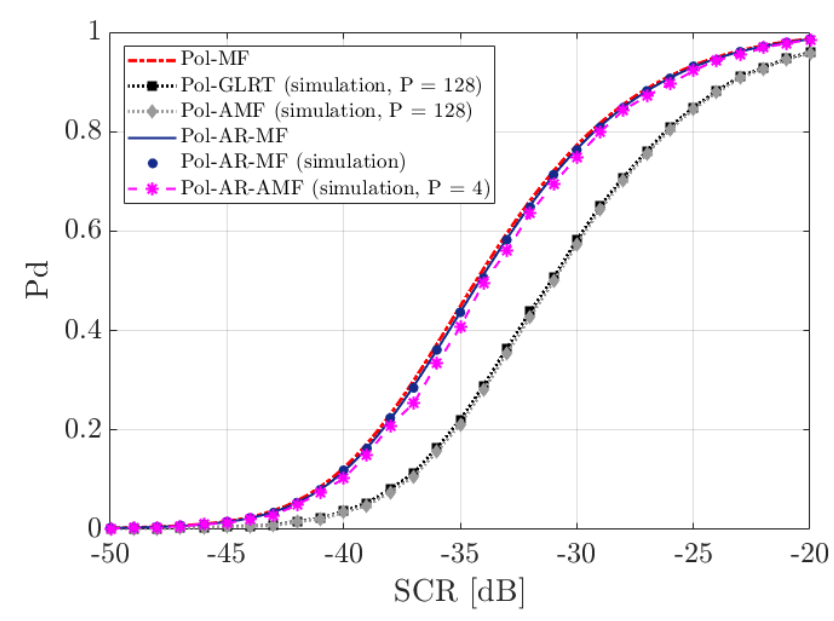

Fig. $6 P_{d}$ versus $\mathrm{SCR}$ for $P_{f a}=10^{-3}, L=2(\mathrm{HH}, \mathrm{VV})$, $M=32$ and $\rho_{t}=0.99$.

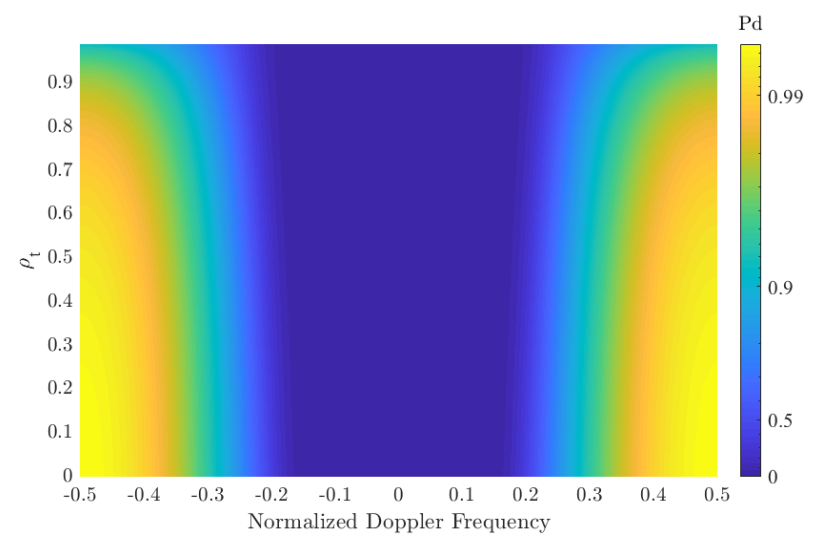

(a)

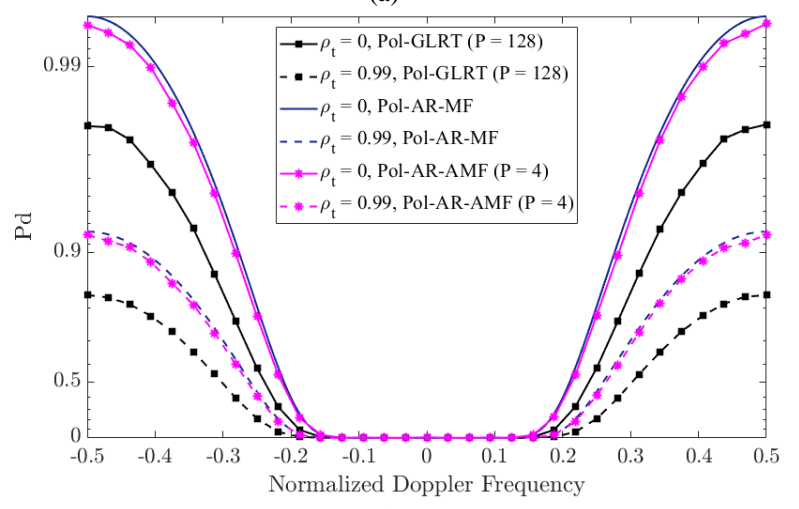

(b)

Fig. $7 P_{d}$ for $\mathrm{SCR}=-35 \mathrm{~dB}, P_{f a}=10^{-3}, L=2(\mathrm{HH}, \mathrm{VV}), M=32$ :

(a) $P_{d}$ for Pol-AR-MF as a function of $f_{d}$ and $\rho_{t}$ (b) $P_{d}$ versus $f_{d}$ for $\rho_{t}=0$ and $\rho_{t}=0.99$.

As it is apparent the performance of all the considered detection schemes degrade since the target echoes at the two co-polarized channels are correlated, so that the target fade average is much less effective than in the former case in which $\rho_{t}=0$. Moreover, the target echoes are also partially cancelled by the clutter cancellation stage of the adaptive detectors which is intended to mitigate polarimetric correlated signals. However, we highlight that the comparative analysis between different adaptive detectors remains unchanged and the PolAR-AMF still outperforms the fully adaptive detectors even operating with a much smaller training data size.

The considerations above are confirmed by Fig. 7 (a-b), where we study the detection performance depending on the selected Doppler frequency and target polarimetric crosscorrelation coefficient. In Fig. 7(a), we consider SCR $=-35$ $\mathrm{dB}$ and $P_{f a}=10^{-3}$, and we report the $P_{d}$ obtained with the proposed Pol-AR-MF for the same scenario used in Fig.6 as a function of $\rho_{t}$ and $f_{d}$. In Fig. 7(b), we compare it with the PolGLRT operated using $P=2 M L=128$ and with the Pol-ARAMF operated using $P=4$, for $\rho_{t}=0$ and $\rho_{t}=0.99$. In both Fig. 7 (a) and (b), a logarithmic scale has been used to enhance the difference at high $P_{d}$ values. As expected, as the target polarimetric correlation coefficient decreases, the cancellation notch obtained with all the considered approaches 
progressively narrows allowing a better discrimination of the target echo against the polarimetric correlated disturbance. However, for both the considered $\rho_{t}$ values, the proposed PolAR-AMF operating with $P=4$ nearly reaches its asymptotic detection performance thus remarkably outperforming the conventional Pol-GLRT. Specifically, it provides a target detection probability improvement up to $20 \%$ in the considered scenario.

\section{CONCLUSION}

In this work, we derived a novel polarimetric adaptive detector based on a multi-channel AR process for the disturbance. The authors derived asymptotic expressions for the performance of the proposed detector and its effectiveness has been investigated via numerical analysis. We have shown that the devised approach is able to improve the target detection capability with respect to traditional detection schemes when both single-pol and multi-pol radar systems are considered. Moreover, based on the adopted parametric method, the proposed detection scheme also allows to relax the requirement on the number of target-free training data and on the computational effort, that are key points in applications where the number of adaptive degrees of freedom is large as well as in heterogeneous environment.

These conclusions are indeed valid when the input disturbance strictly follow the underlying model adopted for the detector design. In real-world scenario this is rarely the case therefore it is of high practical interest to understand the behavior of the proposed detection scheme when applied against disturbance components with diverse spectral characteristics. The interested reader is referred to Part II of this work for a detailed study of these aspects [55].

\section{APPENDIX A \\ DERIVATION OF AR MODEL BASED POLARIMETRIC DETECTOR}

We maximize the numerator of (15) over the unknown target amplitude vector $\boldsymbol{\alpha}$, where the likelihood of $\mathbf{X}_{0}$ under the $H_{1}$ hypothesis is defined in (14). The maximization yields:

$$
\widehat{\boldsymbol{\alpha}}=\underset{\boldsymbol{\alpha}}{\operatorname{argmin}}\left\{\operatorname{tr}\left(\mathbf{S}^{H} \mathbf{P S}\right)-2 \mathfrak{R}\left[\operatorname{tr}\left(\mathbf{X}_{0}^{H} \mathbf{P S}\right)\right]\right\}
$$

Taking into account the definitions of matrices $\mathbf{X}_{0}$ and $\mathbf{S}$ provided in sub-section III-A, we can further develop the expression above as

$$
\begin{aligned}
\widehat{\boldsymbol{\alpha}}=\underset{\boldsymbol{\alpha}}{\operatorname{argmin}} & \left\{\sum_{k=0}^{M-Q} \boldsymbol{\alpha}^{H} \boldsymbol{\Sigma}^{H}(k) \mathbf{P} \boldsymbol{\Sigma}(k) \boldsymbol{\alpha}\right. \\
& \left.-2 \Re\left[\sum_{k=0}^{M-Q} \tilde{\mathbf{x}}_{0}{ }^{H}(k) \mathbf{P} \boldsymbol{\Sigma}(k) \boldsymbol{\alpha}\right]\right\}
\end{aligned}
$$

where $\quad \boldsymbol{\Sigma}(k)=\tilde{\mathbf{t}}(k) \otimes \mathbf{I}_{L}$. By defining $\mathbf{U}=$ $\sum_{k=0}^{M-Q} \boldsymbol{\Sigma}^{H}(k) \mathbf{P} \boldsymbol{\Sigma}(k)$ and $\mathbf{v}=\sum_{k=0}^{M-Q} \tilde{\mathbf{x}}_{0}{ }^{H}(k) \mathbf{P} \boldsymbol{\Sigma}(k)$ becomes

$$
\widehat{\boldsymbol{\alpha}}=\underset{\boldsymbol{\alpha}}{\operatorname{argmin}}\left\{\boldsymbol{\alpha}^{H} \mathbf{U} \boldsymbol{\alpha}-2 \Re[\mathbf{v} \boldsymbol{\alpha}]\right\}
$$

that yields the following ML estimate for the target amplitude vector:

$$
\widehat{\boldsymbol{\alpha}}=\mathbf{U}^{-1} \mathbf{v}^{H}
$$

Eventually, by substituting (41) in (15) we obtain

$$
\begin{aligned}
& \frac{\max _{\boldsymbol{\alpha}}\left\{f_{1}\left(\mathbf{X}_{0} \mid \boldsymbol{\alpha}, \mathbf{R}, \mathbf{A}\right)\right\}}{f_{0}\left(\mathbf{X}_{0} \mid \mathbf{R}, \mathbf{A}\right)} \\
&= 2 \mathfrak{R}\left[\widehat{\boldsymbol{\alpha}}^{H} \mathbf{v}^{H}\right]-\widehat{\boldsymbol{\alpha}}^{H} \mathbf{U} \widehat{\boldsymbol{\alpha}} \\
& H_{1} \\
&= \mathbf{v} \mathbf{U}^{-1} \mathbf{v}^{H} \underset{\ln }{\gtrless}\left(\eta_{0}\right) \\
& H_{0}
\end{aligned}
$$

which, scaled by a constant factor 2, similarly to [52], is converted in (16).

\section{APPENDIX B \\ ML PARAMETER ESTIMATION}

The joint pdf of the secondary data is given by (22). We first look for matrix $\mathbf{A}$ that maximizes (22) or, equivalently, that minimizes $\operatorname{tr}\left(\overline{\mathbf{X}}^{H} \mathbf{P} \overline{\mathbf{X}}\right)$, where $\mathbf{P}=\mathbf{H}^{H} \mathbf{R}^{-1} \mathbf{H}$ and $\mathbf{H}=$ $\left[\begin{array}{ll}-\mathbf{A}^{H} & \mathbf{I}_{L}\end{array}\right]$. To this purpose, we define matrix $\widehat{\mathbf{Q}}=\overline{\mathbf{X}} \overline{\mathbf{X}}^{H}$ that is decomposed in blocks as

$$
\widehat{\mathbf{Q}}=\left[\begin{array}{ll}
\widehat{\mathbf{Q}}_{00} & \widehat{\mathbf{Q}}_{01} \\
\widehat{\mathbf{Q}}_{01}^{H} & \widehat{\mathbf{Q}}_{11}
\end{array}\right]
$$

where $\widehat{\mathbf{Q}}_{00}$ and $\widehat{\mathbf{Q}}_{11}$ are square blocks with dimensions $L(Q-$ $1) \times L(Q-1)$ and $L \times L$ respectively, whereas $\widehat{\mathbf{Q}}_{01}$ is a $L(Q-$ 1) $\times L$ block.

With this definition, we can write:

$$
\begin{array}{r}
\operatorname{tr}\left(\overline{\mathbf{X}}^{H} \mathbf{P} \overline{\mathbf{X}}\right)=\operatorname{tr}\left(\widehat{\mathbf{Q}} \mathbf{H}^{H} \mathbf{R}^{-1} \mathbf{H}\right) \\
=\operatorname{tr}\left(\widehat{\mathbf{Q}}_{00} \mathbf{A} \mathbf{R}^{-1} \mathbf{A}^{H}-\widehat{\mathbf{Q}}_{01} \mathbf{R}^{-1} \mathbf{A}^{H}\right. \\
\left.-\widehat{\mathbf{Q}}_{01}^{H} \mathbf{A} \mathbf{R}^{-1}+\widehat{\mathbf{Q}}_{11} \mathbf{R}^{-1}\right)
\end{array}
$$

and using well known identities for trace derivatives [66], we have:

$$
\frac{\partial}{\partial \mathbf{A}^{*}}\left\{\operatorname{tr}\left(\overline{\mathbf{X}}^{H} \mathbf{P} \overline{\mathbf{X}}\right)\right\}=\widehat{\mathbf{Q}}_{00} \mathbf{A} \mathbf{R}^{-1}-\widehat{\mathbf{Q}}_{01} \mathbf{R}^{-1}
$$

that is equated to zero to obtain the ML estimate of matrix $\mathbf{A}$ :

$$
\widehat{\mathbf{A}}=\widehat{\mathbf{Q}}_{00}^{-1} \widehat{\mathbf{Q}}_{01}
$$


Now, by substituting (46) in (22) we obtain

$$
\begin{aligned}
& \max _{\mathbf{A}}\left\{f_{0}(\overline{\mathbf{X}} \mid \mathbf{R}, \mathbf{A})\right\} \\
& =\left(\pi^{L}|\mathbf{R}|\right)^{-P(M-Q+1)} \exp \left\{-\operatorname{tr}\left(\widehat{\mathbf{H}} \widehat{\mathbf{Q}} \widehat{\mathbf{H}}^{H} \mathbf{R}^{-1}\right)\right\}
\end{aligned}
$$

that can be then maximized with respect to $\mathbf{R}$ thus yielding:

$$
\begin{gathered}
\widehat{\mathbf{R}}=\frac{1}{P(M-Q+1)} \widehat{\mathbf{H}} \widehat{\mathbf{Q}} \widehat{\mathbf{H}}^{H} \\
=\frac{1}{P(M-Q+1)}\left(\widehat{\mathbf{Q}}_{11}-\widehat{\mathbf{Q}}_{01}^{H} \widehat{\mathbf{Q}}_{00}^{-1} \widehat{\mathbf{Q}}_{01}\right)
\end{gathered}
$$

where $\widehat{\mathbf{Q}}_{11}-\widehat{\mathbf{Q}}_{01}^{H} \widehat{\mathbf{Q}}_{00}^{-1} \widehat{\mathbf{Q}}_{01}=\left[\widehat{\mathbf{Q}}^{-1}\right]_{Q, Q}^{-1}$ is the inverse of the last $L \times L$ block on the main diagonal of the inverse of $\widehat{\mathbf{Q}}$. We note that the derived expressions for matrices $\widehat{\mathbf{A}}$ and $\widehat{\mathbf{R}}$ reported in (46) and (48), respectively, are approximations of the actual ML estimates of the AR parameters, given that the pdf in eq. (10) is the approximate (actually conditional) pdf of the data. However, the approximation is quite accurate for large data records, provided that the actual pdf can be well approximated by the conditional pdf [63][64].

\section{APPENDIX C \\ PDF OF $\breve{\mathbf{z}}_{0}$ UNDER THE $H_{0}$ HYPOTHESIS}

In this Appendix, we look for the pdf of vector $\breve{\mathbf{z}}_{0}$, defined as $\breve{\mathbf{z}}_{0}=\mathbf{C}^{H} \mathbf{B}^{H} \mathbf{x}_{0}$, under the $H_{0}$ hypothesis, provided that matrices $\mathbf{B}$ and $\mathbf{C}$ are known. Based on its definition and eq.(10), it is easy to observe that $\left.\breve{\mathbf{z}}_{0}\right|_{H_{0}} \sim \mathcal{C N}\left(\mathbf{0}_{L \times 1}, \mathbf{D}_{0}\right)$, denoting $\mathbf{D}_{0}$ as the disturbance covariance matrix that will be derived in the following .

From (17), we write $\breve{\mathbf{z}}_{0}$ as $\breve{\mathbf{z}}_{0}=\sum_{m=0}^{M-Q} \breve{\mathbf{z}}_{0, m}$, namely as the sum of $M-Q-1$ vectors $\breve{\mathbf{z}}_{0, m}=\mathbf{W}^{-1 / 2} \mathbf{V}^{H}(m) \tilde{\mathbf{x}}_{0}(m)$, each being a $L \times 1$ zero-mean complex Gaussian variable with crosscovariance matrix

$$
\begin{aligned}
& E\left\{\breve{\mathbf{z}}_{0, m} \breve{\mathbf{z}}_{0, n}^{H}\right\} \\
& =\mathbf{W}^{-\frac{1}{2}} \mathbf{V}(m)^{H} E\left\{\tilde{\mathbf{x}}_{0}(m) \tilde{\mathbf{x}}_{0}{ }^{H}(n)\right\} \mathbf{V}(n) \mathbf{W}^{-\frac{1}{2}} \\
& (m, n=0, \ldots, M-Q)
\end{aligned}
$$

where $E\left\{\tilde{\mathbf{x}}_{0}(m) \tilde{\mathbf{x}}_{0}{ }^{H}(n)\right\}$ is a $Q L \times Q L$ block of the disturbance covariance matrix $\mathbf{M}$, whose diagonal is coincident with the main diagonal of $\mathbf{M}$ only if $m=n$.

Using the definition of matrices $\mathbf{V}(m)=\mathbf{P} \boldsymbol{\Sigma}(m)$ and $\mathbf{P}=$ $\mathbf{H}^{H} \mathbf{R}^{-1} \mathbf{H}$, (49) becomes

$$
\begin{aligned}
& E\left\{\breve{\mathbf{z}}_{0, m} \breve{\mathbf{z}}_{0, n}^{H}\right\} \\
& =\mathbf{W}^{-\frac{1}{2}} \boldsymbol{\Sigma}(m)^{H} \mathbf{H}^{H} \mathbf{R}^{-1} \mathbf{H} E\left\{\tilde{\mathbf{x}}_{0}(m) \tilde{\mathbf{x}}_{0}{ }^{H}(n)\right\} \mathbf{H}^{H} \\
& \times \mathbf{R}^{-1} \mathbf{H} \boldsymbol{\Sigma}(n) \mathbf{W}^{-\frac{1}{2}}
\end{aligned}
$$

$$
(m, n=0, \ldots, M-Q)
$$

According to the employed multi-channel AR model in (9), we write the product $\mathbf{H} E\left\{\tilde{\mathbf{x}}_{0}(m) \tilde{\mathbf{x}}_{0}{ }^{H}(n)\right\} \mathbf{H}^{H}$ as

$$
\begin{aligned}
E\left\{\left[\mathbf{H} \tilde{\mathbf{x}}_{0}(m)\right]\left[\tilde{\mathbf{x}}_{0}{ }^{H}(n) \mathbf{H}^{H}\right]\right\} \\
=E\left\{\mathbf{w}(m+Q-1) \mathbf{w}^{H}(n+Q-1)\right\} \\
=\mathbf{R} \delta(m-n)
\end{aligned}
$$

denoting $\delta(\cdot)$ as the Dirac delta function.

Finally, by substituting (51) into (50), we easily obtain

$$
\begin{aligned}
& E\left\{\breve{\mathbf{z}}_{0, m} \breve{\mathbf{z}}_{0, n}^{H}\right\} \\
& = \begin{cases}\mathbf{W}^{-\frac{1}{2}} \boldsymbol{\Sigma}^{H}(m) \mathbf{P} \boldsymbol{\Sigma}(m) \mathbf{W}^{-\frac{1}{2}} & m=n \\
\mathbf{0}_{L} & m \neq n\end{cases}
\end{aligned}
$$

Therefore, the sought covariance matrix $\mathbf{D}_{0}$ is

$$
\mathbf{D}_{0}=\mathbf{W}^{-\frac{1}{2}}\left[\sum_{m=0}^{M-Q} \boldsymbol{\Sigma}^{H}(m) \mathbf{P} \boldsymbol{\Sigma}(m)\right] \mathbf{W}^{-\frac{1}{2}}=2 \mathbf{I}_{L}
$$

Based on (53), we can conclude that $\left.\breve{\mathbf{z}}_{0}\right|_{H_{0}} \sim \mathcal{C N}\left(\mathbf{0}_{L \times 1}, 2 \mathbf{I}_{L}\right)$.

\section{APPENDIX D \\ DERIVATION OF THE ASYMPTOTIC PD FOR FLUCTUATING TARGET MODEL}

In this Appendix, we look for $\operatorname{Prob}\left\{\left\|\breve{\mathbf{z}}_{0}\right\|^{2}>\eta\right\}$, assuming that vector $\breve{\mathbf{z}}_{0}$ is a complex Gaussian random variable with zero-mean vector and covariance matrix $\mathbf{D}_{0}$, i.e. $\left.\breve{\mathbf{z}}_{0}\right|_{H_{1}} \sim \mathcal{C N}\left(\mathbf{0}_{L \times 1}, \mathbf{D}_{0}\right)$. In particular, $\mathbf{D}_{0}=2 \mathbf{I}_{L}+$ $\mathbf{C}^{H} \mathbf{B}^{H}\left(\mathbf{t t}^{H} \otimes \mathbf{M}_{\mathbf{t}}\right) \mathbf{B C}$, where $\mathbf{M}_{t}$ is the target amplitudes covariance matrix. To this end, we follow the main steps of the procedure reported in [67], with reference to the problem under consideration. In order for the results in [67] to be directly applied, we rewrite the test statistic as $T_{P o l-A R-M F}=$ $\mathbf{h}^{H} \mathbf{D}_{0} \mathbf{h}$, where $\mathbf{h}=\left(\mathbf{D}_{0}^{-1 / 2}\right)^{H} \breve{\mathbf{z}}_{0}$ is the whitened version of $\breve{\mathbf{z}}_{0}$, i.e. $\mathbf{h} \sim \mathcal{C} \mathcal{N}\left(\mathbf{0}_{L \times 1}, \mathbf{I}_{L}\right)$.

Let $\lambda_{0} \ldots \lambda_{N-1}$ denote the $N \leq L$ distinct non-zero eigenvalues of matrix $\mathbf{D}_{0}$, each with multiplicity $\mu_{n}, n=0, \ldots$, $N-1$. Without loss of generality, we assume that no eigenvalue of matrix $\mathbf{D}_{0}$ is equal to zero. To see why this is the case, let us consider the eigenvalues decomposition of matrix $\mathbf{D}_{0}$, i.e. $\mathbf{D}_{0}=\mathbf{K} \boldsymbol{\Lambda} \mathbf{K}^{H}$ and let us assume that $\boldsymbol{\Lambda}$ is organized so that $\boldsymbol{\Lambda}=\left[\begin{array}{cc}\overline{\overline{\boldsymbol{\Lambda}}} & \mathbf{0}_{N \times(L-N)} \\ \mathbf{0}_{(L-N) \times N} & \mathbf{0}_{(L-N) \times(L-N)}\end{array}\right]$, where $\overline{\overline{\boldsymbol{\Lambda}}}$ is a $N \times N$ block with the non-zero eigenvalues on its main diagonal. Therefore, the test statistic could be simply reworked as $T_{P o l-A R-M F}=$ $\overline{\overline{\mathbf{h}}}^{H} \overline{\overline{\mathbf{\Lambda}}} \overline{\overline{\mathbf{h}}}$, where $\overline{\overline{\mathbf{h}}}=\boldsymbol{\Theta}^{H}\left(\mathbf{K}^{H} \mathbf{h}\right), \boldsymbol{\Theta}=\left[\begin{array}{lll}\mathbf{I}_{N} & \vdots & \mathbf{0}_{L \times(L-N)}\end{array}\right]^{H}$.

Based on the employed model, we can write the cumulative distribution function (CDF) of $T_{P o l-A R-M F}$ as follows [67]

$$
F_{0}(t)=\frac{1}{2 \pi} \int_{-\infty}^{\infty} \frac{e^{t(j \omega+\beta)}}{\left|\mathbf{I}_{L}+(j \omega+\beta) \boldsymbol{\Lambda}\right|(j \omega+\beta)} d \omega
$$

$$
(\beta>0)
$$


To evaluate this integral, first we resort to a partial fraction expansion for the fraction that appears in (54), obtaining [67]

$$
\begin{aligned}
& \frac{1}{\left|\mathbf{I}_{L}+(j \omega+\beta) \boldsymbol{\Lambda}\right|(j \omega+\beta)}= \\
& \sum_{n=0}^{N-1} \sum_{k=0}^{\mu_{n}-1} \frac{\delta_{k, n}}{\left[\frac{1}{\lambda_{n}}+(j \omega+\beta)\right]^{k+1}}+\frac{1}{(j \omega+\beta)}
\end{aligned}
$$

where the coefficients $\delta_{k, n}$ are given by

$$
\begin{array}{r}
\delta_{k, n}=\left.\frac{\prod_{j=0}^{N-1} \lambda_{j}^{-\mu_{j}}}{\Gamma\left(\mu_{n}-k\right)} y_{n}^{\left(\mu_{n}-k-1\right)}(s)\right|_{s=-\frac{1}{\lambda_{n}}} \\
\left(n=0, \ldots, N-1, k=0, \ldots, \mu_{n}-1\right)
\end{array}
$$

being $y_{n}^{\left(\mu_{n}-k-1\right)}(s)$ the $\left(\mu_{n}-k-1\right)$-th derivative of $y_{n}(s)$, defined as

$$
y_{n}(s)=\prod_{\substack{j=0 \\ j \neq n}}^{N}\left(\zeta_{j}+s\right)^{-\mu_{j}}
$$

with $\zeta_{n}=\frac{1}{\lambda_{n}}, n=0, \ldots, N-1, \zeta_{N}=0$ and $\mu_{N}=1$.

One can evaluate the coefficient $y_{n}^{\left(\mu_{n}-k-1\right)}$ in $\delta_{k, n}$ differentiating the logarithm of $y_{n}(s)$, i.e. $\frac{d}{d s} \log \left[y_{n}(s)\right]=$ $\frac{1}{y_{n}(s)} y_{n}^{(1)}(s)$, that yields

$$
y_{n}^{(1)}(s)=-y_{n}(s) \sum_{\substack{j=0 \\ j \neq n}}^{N} \mu_{j}\left(\zeta_{j}+s\right)^{-1}
$$

Subsequently, Leibniz's rule for differentiation of products may be applied to evaluate the $p$-th order derivative.

$$
\begin{aligned}
y_{n}^{(p)}(s)= & \frac{d^{p-1}}{d s^{p-1}} y_{n}^{(1)}(s) \\
& =-\sum_{r=0}^{p-1}\left(\begin{array}{c}
p-1 \\
r
\end{array}\right) y_{n}^{(r)}(s) \\
& \times \frac{d^{p-1-r}}{d s^{p-1-r}}\left[\sum_{\substack{j=0 \\
j \neq n}}^{N} \mu_{j}\left(\zeta_{j}+s\right)^{-1}\right]
\end{aligned}
$$

Thus, the required quantities are obtained using the following recursion formula

$$
\begin{aligned}
& \left.y_{n}^{(p)}(s)\right|_{s=-\zeta_{n}} \\
= & \left.\sum_{r=0}^{p-1} \sum_{\substack{j=0 \\
j \neq n}}^{N} \mu_{j}\left(\begin{array}{c}
p-1 \\
r
\end{array}\right) \frac{(-1)^{p-r} \Gamma(p-r)}{\left(\zeta_{j}-\zeta_{n}\right)^{p-r}} y_{n}^{(r)}(s)\right|_{s=-\zeta_{n}}
\end{aligned}
$$

$(p \geq 1)$

$$
\left.y_{n}^{(0)}(s)\right|_{s=-\zeta_{n}}=y_{n}\left(-\zeta_{n}\right)=\prod_{\substack{j=0 \\ j \neq n}}^{N}\left(\zeta_{j}-\zeta_{n}\right)^{-\mu_{j}}
$$

By substituting the results in (60) into (56), the coefficients $\delta_{k, n}$ are obtained and can be used to evaluate the partial fraction expansion in (55). Finally, this result can be employed in (54) to solve the integral and to obtain, after some calculations

$$
F_{0}(t)=1+\sum_{n=0}^{N-1} \sum_{k=0}^{\mu_{n}-1} \frac{e^{\left(-\frac{t}{\lambda_{n}}\right)} t^{k}}{\Gamma(k+1)} \delta_{k, n}
$$

$$
(t \geq 0)
$$

From the CDF in (61), the derivation of the $P_{d}$ expression in (32) is straightforward

$$
P_{d}=1-F_{0}(\eta)=\sum_{n=0}^{N-1} \sum_{k=0}^{\mu_{n}-1} \frac{-e^{\left(-\frac{\eta}{\lambda_{n}}\right)} \eta^{k}}{\Gamma(k+1)} \delta_{k, n}
$$

In the special case of only one eigenvalue $(N=1)$ equal to $\lambda_{0}$ with multiplicity $\mu_{0}=L$, the residues in (56) can be simplified as

$$
\delta_{k, 0}=-\lambda_{0}^{-k}
$$

from which the $P_{d}$ in (33) is obtained.

In the dual special case when no eigenvalue is repeated, namely when $N=L$ and $\mu_{n}=1, n=0, \ldots, N-1$ the residues in (56) can be simplified as

$$
\delta_{0, n}=-\lambda_{n} \prod_{\substack{j=0 \\ j \neq n}}^{L-1}\left(\lambda_{n}-\lambda_{j}\right)^{-1}
$$

Using (64), we easily obtain the $P_{d}$ expression in (35).

\section{ACNOWLEDGEMENT}

The authors gratefully acknowledge the anonymous reviewers and the associate editor for their constructive comments on the manuscript.

\section{REFERENCES}

[1] D. Giuli, "Polarization diversity in radars," in Proceedings of the IEEE, vol. 74, no. 2, pp. 245-269, Feb. 1986.

[2] L. M. Novak, M. B. Sechtin and M. J. Cardullo, "Studies of target detection algorithms that use polarimetric radar data," in IEEE Trans. on Aerospace and Electronic Systems, 25 (2), pp. 150-165, Mar. 1989.

[3] W. Boerner and Y. Yamaguchi, "A state-of-the-art review in radar polarimetry and its applications in remote sensing," in IEEE Aerospace and Electronic Systems Magazine, vol. 5, no. 6, pp. 3-6, June 1990.

[4] H. R. Park, J. Li, H. Wang, "Polarization-space-time domain generalized likelihood ratio detection of radar targets", Signal 
Processing, vol. 41, pp. 153-164, 1995.

[5] D. Pastina, P. Lombardo and T. Bucciarelli, "Adaptive polarimetric target detection with coherent radar. I. Detection against Gaussian background," in IEEE Transactions on Aerospace and Electronic Systems, vol. 37, no. 4, pp. 1194-1206, Oct. 2001.

[6] A. De Maio, G. Ricci, "A polarimetric adaptive matched filter", Signal Processing, vol. 81, no. 12, pp. 2583-2589, Dec. 2001.

[7] P. Lombardo, D. Pastina and T. Bucciarelli, "Adaptive polarimetric target detection with coherent radar. II. Detection against non-Gaussian background," in IEEE Trans. on AES, 37(4), pp. 1207-1220, Oct. 2001.

[8] G. Alfano, A. De Maio and E. Conte, "Polarization diversity detection of distributed targets in compound-Gaussian clutter," in IEEE Trans. on AES, vol. 40, no. 2, pp. 755-765, April 2004.

[9] M. Hurtado and A. Nehorai, "Polarimetric Detection of Targets in Heavy Inhomogeneous Clutter," in IEEE Transactions on Signal Processing, vol. 56, no. 4, pp. 1349-1361, April 2008.

[10] S. Gogineni and A. Nehorai, "Polarimetric MIMO Radar With Distributed Antennas for Target Detection," in IEEE Transactions on Signal Processing, vol. 58, no. 3, pp. 1689-1697, March 2010.

[11] A. P. G. Ariza and R. S. Thomä, "Polarimetric ultrawideband MIMO radar for security check points: Detecting and classifying suspects carrying wires," 2012 6th European Conference on Antennas and Propagation (EUCAP), Prague, 2012, pp. 1733-1736.

[12] J. W. F. Goddard and S. M. Cherry, "The ability of dual-polarization radar (copolar linear) to predict rainfall rate and microwave attenuation," in Radio Science, 19 (01), pp. 201-208, Jan.-Feb. 1984.

[13] F. S. Marzano, G. Botta and M. Montopoli, "Iterative Bayesian Retrieval of Hydrometeor Content From X-Band Polarimetric Weather Radar," in IEEE Transactions on Geoscience and Remote Sensing, vol. 48, no. 8, pp. 3059-3074, Aug. 2010.

[14] Islam, T., M.A. Rico-Ramirez, D. Han, and P.K. Srivastava,:"Artificial intelligence techniques for clutter identification with polarimetric radar signatures". Atmospheric Research, vol. 109-110, pp. 95-113 2012.

[15] H. A. Zebker and J. J. Van Zyl, "Imaging radar polarimetry: a review," in Proceedings of the IEEE, vol. 79, no. 11, pp. 1583-1606, Nov. 1991.

[16] L. M. Novak and M. C. Burl, "Optimal speckle reduction in polarimetric SAR imagery," in IEEE Transactions on Aerospace and Electronic Systems, vol. 26, no. 2, pp. 293-305, March 1990.

[17] D. Pastina, P. Lombardo, A. Farina, P. Daddi, "Super-resolution of polarimetric SAR images of ship targets", Signal Processing, vol. 83, no. 8, pp. 1737-1748, 2003

[18] M. Martorella, J. Palmer, F. Berizzi, B. Haywood and B. Bates, "Polarimetric ISAR autofocusing," in IET Signal Processing, vol. 2, no. 3, pp. 312-324, September 2008.

[19] L. Pallotta and D. Orlando, "Polarimetric Covariance Eigenvalues Classification in SAR Images," in IEEE Geoscience and Remote Sensing Letters, vol. 16, no. 5, pp. 746-750, May 2019.

[20] D. Tahmoush, J. Silvious, "Radar Measurement of Human Polarimetric Micro-Doppler", Hindawi Journal of Electrical and Computer Engineering, vol. 2013.

[21] R. Rytel-Andrianik et al., "Simple X-band polarimetric micro-Doppler analyses of ground moving targets," 2015 Signal Processing Symposium (SPSympo), Debe, 2015, pp. 1-4.

[22] O. A. Krasnov and A. G. Yarovoy, "Polarimetric micro-Doppler characterization of wind turbines," 2016 10th European Conference on Antennas and Propagation (EuCAP), Davos, 2016, pp. 1-5.

[23] B. K. Kim, H. Kang and S. Park, "Experimental Analysis of Small Drone Polarimetry Based on Micro-Doppler Signature," in IEEE Geoscience and Remote Sensing Letters, vol. 14, no. 10, pp. 1670-1674, Oct. 2017

[24] F. Colone and P. Lombardo, "Polarimetric passive coherent location," in IEEE Transactions on Aerospace and Electronic Systems, vol. 51, no. 2, pp. 1079-1097, April 2015.

[25] F. Colone and P. Lombardo, "Non-coherent adaptive detection in passive radar exploiting polarimetric and frequency diversity," in IET Radar, Sonar \& Navigation, vol. 10, no. 1, pp. 15-23, 12016.
[26] F. Filippini, F. Colone, D. Cristallini and G. Bournaka, "Experimental results of polarimetric detection schemes for DVB-T-based passive radar," in IET Radar, Sonar \& Navigation, vol. 11, no. 6, pp. 883-891, 62017.

[27] J. You, X. Wan, Y. Fu and G. Fang, "Experimental study of polarisation technique on multi-FM-based passive radar," in IET Radar, Sonar \& Navigation, vol. 9, no. 7, pp. 763-771, 82015.

[28] I. Son and B. Yazici, "Passive polarimetrie multistatic radar for ground moving target," 2016 IEEE Radar Conference (RadarConf), Philadelphia, PA, 2016, pp. 1-6.

[29] A. De Maio and M. Greco, Modern Radar Detection Theory, SciTech Pub., 2016

[30] J. P. Burg, D. G. Luenberger and D. L. Wenger, "Estimation of structured covariance matrices," in Proceedings of the IEEE, vol. 70, no. 9, pp. 963-974, Sept. 1982.

[31] D. R. Fuhrmann, "Application of Toeplitz covariance estimation to adaptive beamforming and detection," in IEEE Transactions on Signal Processing, vol. 39, no. 10, pp. 2194-2198, Oct. 1991.

[32] R. Nitzberg, "Application of Maximum Likelihood Estimation of Persymmetric Covariance Matrices to Adaptive Processing," in IEEE Transactions on Aerospace and Electronic Systems, vol. AES-16, no. 1, pp. 124-127, Jan. 1980.

[33] L. Cai and H. Wang, "A persymmetric multiband GLR algorithm," in IEEE Transactions on Aerospace and Electronic Systems, vol. 28, no. 3, pp. 806-816, July 1992.

[34] G. Pailloux, P. Forster, J. Ovarlez and F. Pascal, "Persymmetric Adaptive Radar Detectors," in IEEE Transactions on Aerospace and Electronic Systems, vol. 47, no. 4, pp. 2376-2390, October 2011.

[35] E. Conte, A. De Maio, "Exploiting persymmetry for CFAR detection in compound-Gaussian clutter", IEEE Transactions on Aerospace and Electronic Systems, vol. 39, no. 2, pp. 719-724, Apr. 2003.

[36] A. Aubry , A. De Maio, L. Pallotta, and A. Farina, "Maximum likelihood estimation of a structured covariance matrix with a condition number constraint," IEEE Transactions on Signal Processing, vol. 60, no. 6, pp. $3004-3021$, Mar. 2012

[37] B. Kang , V. Monga, and M. Rangaswamy, " Rank-constrained maximum likelihood estimation of structured covariance matrices," IEEE Trans. on AES, vol. 50, no. - 1, pp. 501-515, Jan. 2014.

[38] J. J. Shynk, Probability Random Variables and Random Processes: Theory and Signal Processing Applications, New York, NY, USA:Wiley, 2012.

[39] S. Haykin, A. Steinhardt, Adaptive radar detection and estimation, New York : Wiley, 1992.

[40] H. Krim and M. Viberg, "Two decades of array signal processing research: the parametric approach," in IEEE Signal Processing Magazine, vol. 13, no. 4, pp. 67-94, July 1996.

[41] D. E. Bowyer, P. K. Rajasekaran and W. W. Gebhart, "Adaptive Clutter Filtering Using Autogressive Spectral Estimation," in IEEE Transactions on Aerospace and Electronic Systems, vol. AES-15, no. 4, pp. 538-546, July 1979.

[42] P. Metford, S. Haykin and D. Taylor, "An innovations approach to discrete-time detection theory (Corresp.)," in IEEE Transactions on Information Theory, vol. 28, no. 2, pp. 376-380, March 1982.

[43] S. M. Kay and V. Nagesha, "Maximum likelihood estimation of signals in autoregressive noise," in IEEE Transactions on Signal Processing, vol. 42, no. 1, pp. 88-101, Jan. 1994

[44] A. Sheikhi, M. M. Nayebi and M. R. Aref, "Adaptive detection algorithm for radar signals in autoregressive interference," in IEE Proceedings - Radar, Sonar and Navigation, vol. 145, no. 5, pp. 309314, Oct. 1998.

[45] X. Shuai, L. Kong, J. Yang, "AR-model-based adaptive detection of range-spread targets in compound Gaussian clutter", Signal Processing, vol. 91, pp. 750-758, Aug. 2011.

[46] G. Alfano, A. De Maio and A. Farina, "Model-based adaptive detection of range-spread targets," in IEE Proceedings - Radar, Sonar and Navigation, vol. 151, no. 1, pp. 2-10, 14 Feb. 2004. 
[47] J. H. Michels, P. Varshney and D. Weiner, "Multichannel signal detection involving temporal and cross-channel correlation," in IEEE Transactions on Aerospace and Electronic Systems, vol. 31, no. 3, pp. 866-880, July 1995.

[48] A. L. Swindlehurst and P. Stoica, "Maximum likelihood methods in radar array signal processing," in Proceedings of the IEEE, vol. 86, no. 2, pp. 421-441, Feb. 1998.

[49] J. R. Roman, M. Rangaswamy, D. W. Davis, Qingwen Zhang, B. Himed and J. H. Michels, "Parametric adaptive matched filter for airborne radar applications," in IEEE Trans. on AES, 36(2), pp. 677-692, April 2000.

[50] Jian Li, Guoqing Liu, Nanzhi Jiang, P. Stoica, "Moving target feature extraction for airborne high-range resolution phased-array radar," in IEEE Trans. on Signal Processing, 49(2), pp. 277-289, Feb. 2001.

[51] R. Klemm, Applications of space-time adaptive processing, The Institution of Electrical Engineers, 2004

[52] K. J. Sohn, H. Li and B. Himed, "Parametric GLRT for Multichannel Adaptive Signal Detection," in IEEE Transactions on Signal Processing, vol. 55, no. 11, pp. 5351-5360, Nov. 2007.

[53] C. Hao, S. Gazor, D. Orlando, G. Foglia and J. Yang, "Parametric space-time detection and range estimation of a small target," in IET Radar, Sonar \& Navigation, vol. 9, no. 2, pp. 221-231, 22015.

[54] Y. Gao, H. Li and B. Himed, "Adaptive Subspace Tests for Multichannel Signal Detection in Auto-Regressive Disturbance," in IEEE Trans. on Signal Processing,66(21), pp. 5577-5587, Nov. 2018.

[55] F. Colone and F. Filippini: "Auto-regressive model based Polarimetric Adaptive Detection Scheme. Part II: Performance Assessment under Spectral Model Mismatch," accepted for pubblication in IEEE Transactions on Aerospace and Electronic Systems, COMPANION PAPER

[56] F. Filippini and F. Colone,: "Polarimetric Detection Scheme for Passive Radar based on a 2D Auto-Regressive Disturbance Model", 2019 International Radar Conference (RADAR 2019), Toulon (France).

[57] A. Aubry, V. Carotenuto, A. De Maio and L. Pallotta, "Assessing Reciprocity in Polarimetric SAR Data," in IEEE Geoscience and Remote Sensing Letters, vol. 17, no. 1, pp. 87-91, Jan. 2020.

[58] S. Haykin, B. W. Currie and S. B. Kesler, "Maximum-entropy spectral analysis of radar clutter," in Proceedings of the IEEE, vol. 70, no. 9, pp. 953-962, Sept. 1982.

[59] P. Thomas and S. Haykin, "Stochastic modelling of radar returns," in IEE Proceedings F-Communications, Radar and Signal Processing, vol. 133, no. 5, pp. 476-481, August 1986.

[60] M. Greco, F. Bordoni and F. Gini, "X-band sea-clutter nonstationarity: influence of long waves," in IEEE Journal of Oceanic Engineering, vol. 29, no. 2, pp. 269-283, April 2004.

[61] M. Pinsky, J. Figueras i Ventura, T. Otto, A. Sterkin, A. Khain and H. W. J. Russchenberg, "Application of a Simple Adaptive Estimator for an Atmospheric Doppler Radar," in IEEE Transactions on Geoscience and Remote Sensing, vol. 49, no. 1, pp. 115-127, Jan. 2011.

[62] S. M. Kay, Modern Spectral Estimation, Theory and Application. Englewood Cliffs, NJ: Prentice Hall, 1988.

[63] G. Box, and G. Jenkins, Time Series Analysis: Time Series Analysis, Forecasting and Control, Holden-Day Inc, 1970.

[64] S. M. Kay, Fundamentals of Statistical Signal Processing: Detection Theory, vol. II. Englewood Cliffs, NJ, USA: Prentice-Hall, 1993.

[65] P. Swerling, "Probability of detection for fluctuating targets," in IRE Trans. on Information Theory, vol. 6, no. 2, pp. 269-308, Apr. 1960.

[66] A. Hjorungnes and D. Gesbert, "Complex-Valued Matrix Differentiation: Techniques and Key Results," in IEEE Transactions on Signal Processing, vol. 55, no. 6, pp. 2740-2746, June 2007.

[67] T. Y. Al-Naffouri, M. Moinuddin, N. Ajeeb, B. Hassibi and A. L. Moustakas, "On the Distribution of Indefinite Quadratic Forms in Gaussian Random Variables," in IEEE Transactions on Communications, vol. 64, no. 1, pp. 153-165, Jan. 2016.

[68] H. Hytti, R. Takalo, and H. Ihalainen, "Tutorial on multivariate autoregressive modelling," Journal of clinical monitoring and computing, 20(2):101-108, 2006.

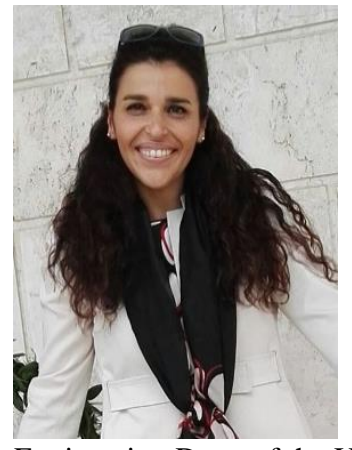

Fabiola Colone received the laurea degree (B.S.+M.S.) in Telecommunications Engineering and the Ph.D. degree in Remote Sensing from Sapienza University of Rome, Italy, in 2002 and 2006, respectively. She joined the DIET Dept. (formerly INFOCOM) of Sapienza University of Rome as a Research Associate in January 2006. From December 2006 to June 2007, she was a Visiting Scientist at the Electronic and Electrical Engineering Dept. of the University College London, London, U.K. She is currently an Associate Professor at the Faculty of Information Engineering, Informatics, and Statistics of Sapienza University of Rome.

The majority of Dr. Colone's research activity is devoted to radar systems and signal processing. She has been involved, with scientific responsibility roles, in research projects funded by the European Commission, the European Defence Agency, the Italian Space Agency, the Italian Ministry of Research, and the radar industry. Her research has been reported in over 120 publications in international technical journals, book chapters, and conference proceedings. Dr. Colone has been co-recipient of the 2018 Premium Award for Best Paper in IET Radar, Sonar \& Navigation.

Since 2017 she is member of the Board of Governors of the IEEE Aerospace and Electronic System Society (AESS) in which she is currently serving as Vice-President for Member Services, and Editor in Chief for the IEEE AESS QEB Newsletters. She is IEEE Senior Member from 2017 and member of the IEEE AESS Radar System Panel from 2019. Dr. Colone is Associate Editor for the IEEE Transactions on Signal Processing and member of the Editorial Board of the Int. Journal of Electronics and Communications (Elsevier). She served in the technical committee of many international conferences. She was in the organizing committee, as the Student Forum Co-Chair, of the IEEE 2008 Radar Conference (Rome, Italy), and she is currently in the organizing committee, as Special Sessions Co-Chair, of the IEEE 2020 Radar Conference (Florence, Italy). She is also Technical co-Chair of the IEEE Radar Conference 2021 (Atlanta, USA).

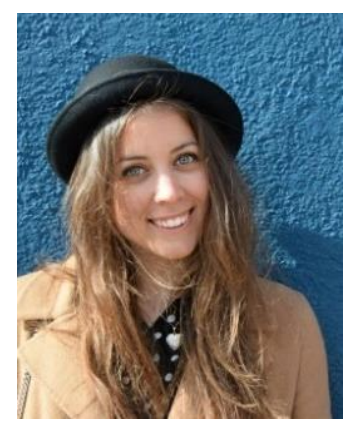

Francesca Filippini (S'17-M'20) received her M.Sc. degree (cum Laude) in Communication Engineering and the $\mathrm{PhD}$ degree in Radar and Remote Sensing, from Sapienza University of Rome, in 2016 and 2020, respectively. From January to May 2016, she has been working on her Master Thesis at Fraunhofer Institute FHR, as an intern of the Passive Radar and Anti-Jamming Techniques Department. She is currently a PostDoctoral Researcher with the DIET Dept. of Sapienza University of Rome.

Dr. Filippini received the 2018 Premium Award for the Best Paper in IET Radar, Sonar \& Navigation, the Best Paper Award at the 2019 Int. Radar Conference, the second Best Student Paper Award at the 2018 IEEE Radar Conference and the Best Paper Award at the 2017 GTTI Workshop on Radar and Remote Sensing. She is a Member of the IEEE AESS Board of Governors, where she is currently serving as Graduate Student Representative, and Chair of the AESS Professional Networking and Mentoring Program. 